\title{
NeuroRegulation
}

\section{Proceedings of the 2019 ISNR Conference}

\author{
Selected Abstracts of Conference Presentations at the 2019 International Society for Neurofeedback \\ and Research (ISNR) 27th Conference, Denver, Colorado, USA
}

Citation: International Society for Neurofeedback and Research. (2019). Proceedings of the 2019 ISNR Conference: Keynotes, Plenary Sessions, and Poster Presentations. NeuroRegulation, 6(4), 197-224. https://doi.org/10.15540/nr.6.4.197

Copyright: @ 2019. ISNR. This is an Open Access article distributed under the terms of the Creative Commons Attribution License (CC-BY).

\section{KEYNOTE PRESENTATIONS}

\section{Towards Enhancing Neurofeedback Research with Advances in Real-time Monitoring of the Central, Autonomic, and Enteric Nervous Systems \\ Todd Coleman \\ University of California, San Diego, California, USA}

In this talk, we will highlight recent technological and methodological advances in developing unobtrusive wearable technologies that can monitor many aspects of the central, autonomic, and enteric nervous systems. As an example, we will discuss our research group's development of stretchable electronic biosensors that can be embedded within routinely used medical adhesives and applied to any part of the body for wireless monitoring. We will also discuss methodological developments to robustly track the dynamic interplay between the central, autonomic, and enteric nervous systems with statistical signal processing of electroencephalography (EEG), electrocardiography (ECG), and electrogastrography (EGG) signals, respectively. We will further highlight recent findings in the research literature that showcase their interrelationship in health and disease and how their readouts have created new opportunities to advance neurofeedback therapies. With an interest in exploring beyond what is currently deployed or known in the neurorehabilitation field, we will highlight recent advances with the high-resolution EGG, which involves placing an array of electrical sensors on the abdomen to produce an "EKG of the digestive system." To elucidate what may be actionable, we will discuss an ongoing clinical study involving neurofeedback that exploits the dynamics of the gutbrain axis. We will conclude with a vision of how the rapid advances in multiorgan physiology research, technology miniaturization, data science, and design principles create a bright future for the field of neurofeedback.

\section{Understanding (and Improving) \\ Neurofeedback Efficacy: A Multidisciplinary Endeavor \\ Eddy Davelaar \\ Birkbeck, University of London, Bloomsbury, London, England}

In this talk, I will present our theoretical (and empirical) work aimed at answering the question "How does neurofeedback work?". The research is guided by the recently formulated multistage theory of neurofeedback learning (Davelaar, 2018). The following examples will be explored. First, giving instructions to trainees has typically been discouraged in the literature on grounds that trainees are inconsistent in their strategy use, they have no knowledge about their strategies, and strategies do not work for everyone. Yet we find that in the case of frontal alpha upregulation, converging verbal reports emerged that are amenable for guided neurofeedback (Davelaar, Barnby, Almasi, \& Eatough, 2018). These results require using the explicitation interview as a research methodology followed by a cognitive classification of the reports. Second, the typical study has a pre- and posttraining session of cognitive tests (and perhaps even a qEEG). I will demonstrate that analyzing the cognitive data beyond the superficial averaged response time and accuracy uncovers cognitive changes that are specific to certain theoretically motivated EEG frequencies (Davelaar, 2017). Thus, sensorimotor rhythm (SMR) training and midfrontal theta upregulation show differential influences on first- and second-order attention. In addition, upregulating frontal alpha or midfrontal theta have opposite effects on information processing that are referred to as nondecision processes, such as feature extraction. Finally, I will present a roadmap for how pre- and posttraining qEEG (and intratraining EEG recordings) can be analyzed to investigate the effects on brain circuitry, which after all is the main aim of neurofeedback training. In sum, the development of an explicit theoretical model opens the door for the field of neurofeedback to adopt formal qualitative and quantitative methodologies from cognitive science 
and tackle research questions that were hitherto beyond the field's reach.

\section{References}

Davelaar, E. J. (2017). Testing the specificity of EEG neurofeedback training on first- and second-order measures of attention. In D. Schmorrow \& C. Fidopiastis (Eds.), AC 2017: Augmented Cognition. Neurocognition and Machine Learning. Lecture Notes in Computer Science (Vol. 10284, pp. 19-27). Switzerland: Springer. https://doi.org/10.1007 /978-3-31958628-1_2

Davelaar, E. J. (2018). Mechanisms of neurofeedback: A computation-theoretic approach. Neuroscience, 378, 175-188. https://doi.org/10.1016/j.neuroscience.2017.05.052

Davelaar, E. J., Barnby, J. M., Almasi, S., \& Eatough, V. (2018). Differential subjective experiences in learners and nonlearners in frontal alpha neurofeedback: piloting a mixedmethod approach. Frontiers in Human Neuroscience, 12, 402. https://doi.org/10.3389/fnhum.2018.00402

\section{Outcomes of Double-Blind Randomized Clinical Trial of Neurofeedback for Attention- Deficit/Hyperactivity Disorder \\ Roger deBeus \\ University of North Carolina, Asheville, North Carolina, USA \\ For the Neurofeedback Collaborative Group}

Objective. To determine whether neurofeedback (NF) has a lasting and specific benefit on inattention in attention-deficit/hyperactivity disorder (ADHD) beyond artifact inhibition and other nonspecific effects such as advice on sleep hygiene, nutrition, coaching, etc. Unblinded randomized clinical trials (RCTs) have shown encouraging results, but small blinded, flawed RCTs have not.

Method. Children age 7-10 (age 8.4 \pm 1.14 years; $78 \%$ male, $76 \%$ Caucasian, 13\% Latino, $8 \%$ African American, 4\% Asian) at two sites were randomly assigned in a 3:2 ratio to 38 sessions of active neurofeedback (three times per week) using the Lubar-Monastra method to downtrain theta-beta ratio (TBR) vs. sham neurofeedback of equal duration, frequency, and intensity. Primary outcome (parentand teacher-rated inattentive symptoms) was analyzed by a linear mixed model with time, treatment, time $X$ treatment interaction, site, and site $X$ treatment interaction entered.

Results. 329 children were screened, resulting in 144 randomized, with 142 in the intent-to-treat analysis. Children were diagnosed as inattentive (36\%) or combined type (64\%) ADHD; $69 \%$ had comorbid diagnoses (50\% oppositional defiant disorder, $21 \%$ internalizing disorders). There were 10 (7\%) dropouts from treatment. Treatment fidelity was good $(98 \%$ by trainer report, $84 \%$ by independent fidelity rater). Across treatment arms, there were 94 adverse events possibly related to treatment (e.g., eye pain, irritability, oppositionality, crying, self-injury, headache, depression, skin irritation). Blinded guesses as to sham treatment assignment were correct $7 \%$ of the time by children, $24 \%$ by trainers, and $25 \%$ by parents. Substantial improvements on the primary outcome measure, parent- and teacherrated inattention, were found, with a large pre- and posttreatment effect size $(d=1.1) ; 60 \%$ of the children responded to treatment, and, most importantly, clinical benefit appears to be maintained at 13-month follow-up. However, these benefits were seen in both groups and not significantly different between the neurofeedback and control group at treatment end. Nonetheless, preliminary results at 13-month follow-up demonstrate the neurofeedback group showed further improvements on inattention, whereas the control group remained stable with a medium effect size between-group difference. Complete statistical results on the full sample at 13month follow-up will be presented.

Conclusion. The multimodal nature of the treatment-including supportive coaching, advice on sleep hygiene and nutrition, and practice focusing on a nonentertaining screen-likely contributed to the control group's large improvement, resulting in the lack of short-term difference. In fact, some evidence was found that the control condition might not have been fully inert, since some above-chance-level learning was observed in the control group, although that could not explain all the observed benefit for controls. Further analyses and study will have to focus on explaining the good response in the control group; however, preliminary results suggest a medium specific delayed "sleeper" effect of NF at 13month follow-up.

\section{Epilepsy and the Foundation of NF Jay Gunkelman \\ Brain Science International, San Ramon, California, USA}

In the beginning there were those doing "state-based" EEG training like Joe Kamiya and Elmer Green, and those doing "clinical" work with epilepsy associated largely with Barry Sterman or Nils Birbaumer. ADHD and other applications came later...but the scientific proof-level work in epilepsy was quite impressive even in the mid-1970s. Despite the efficacy proofs, most NF practitioners today do not work with epilepsy as a primary indication for clinical work.... At least not knowingly. 
However, approximately $20 \%$ of those with ADHD and from 40 to $60 \%$ of those with ASD have "unexpected" epileptiform discharges or paroxysms. These clients still need our help. There are also many thousands with intractable epilepsy.

A series of severe intractable epileptic cases illustrates the life-changing nature of applying NF clinically. Very current publications on the efficacy of treating psychiatric clients who have epileptiform activity but no seizure history will be shared, as will publications challenging the standard of practice in psychiatry in treating these clients without reviewing the EEG.

\section{References}

Boutros, N. (2009). Epileptiform discharges in psychiatric patients: A controversy in need of resurrection. Clinical EEG and Neuroscience, 40(4), 239-244. https://doi.org/10.1177 $/ 155005940904000406$

Boutros, N. N., Kirollos, S. B., Pogarell, O., \& Gallinat, J. (2014). Predictive value of isolated epileptiform discharges for a favorable therapeutic response to antiepileptic drugs in nonepileptic psychiatric patients. Journal of Clinical Neurophysiology, 31(1), 21-30. https://doi.org/10.1097 MNP.0000000000000023

Millichap, J. G., Millichap, J. J., \& Stack, C. V. (2011). Utility of the electroencephalogram in attention deficit hyperactivity disorder. Clinical EEG and Neuroscience, 42(3), 180-184. https://doi.org/10.1177/155005941104200307

Swatzyna, R. J., Tarnow, J. D., Proler, M. L., Roark, A. J., Maclnerney, E. K., \& Kozlowski, G. P. (2017). Retrospective analysis of nonepileptic patients with isolated epileptiform discharges treated with anticonvulsants. Clinical EEG and Neuroscience, 48(5), 322-326. https://doi.org/10.1177 /1550059417695896

Swatzyna, R. J., Tarnow, J. D., Turner, R. P., Roark, A. J. MacInerney, E. K., \& Kozlowski, G. P. (2017). Integration of EEG into psychiatric practice: A step toward precision medicine for autism spectrum disorder. Journal of Clinical Neurophysiology, 34(3), 230-235. https://doi.org/10.1097 WNP.0000000000000365

\section{Critical Brain Dynamics: A Novel Framework for Assessing and Regulating Brain Dynamics Oren Shriki \\ Ben-Gurion University of the Negev, Beersheba, Israel}

The critical brain hypothesis proposes that our brain is poised close to the border between two qualitatively different dynamical states. Whereas subcritical dynamics are characterized by premature termination of activity propagation, supercritical dynamics are associated with runaway excitation. The talk will review evidence from recent years that supports this hypothesis and will introduce the concept of neuronal avalanches, spatiotemporal cascades of activity whose sizes obey a power-law distribution. They are observed in a wide range of experiments from small- scale cortical networks to large-scale human EEG and are considered as evidence for critical brain dynamics. The avalanche analysis provides novel qEEG measures which reflect the neural gain and are sensitive to changes in the balance of excitatory and inhibitory processes. Consequently, deviations from critical dynamics could serve as biomarkers for disorders associated with altered balance. For example, in sleep deprivation and in epilepsy the system tends towards supercritical dynamics, whereas in disorders of consciousness the system displays subcritical dynamics. EEG-based measures of criticality can also be used as parameters for neurofeedback. In particular, our lab developed an EEG-based neurofeedback system that evaluates subjects' neural gain using online avalanche analysis and reflects it by means of a video game. Our preliminary results indicate that subjects can regulate their neural gain.

\section{References}

Arviv, O., Medvedovsky, M., Sheintuch, L., Goldstein, A., \& Shriki, O. (2016). Deviations from critical dynamics in interictal epileptiform activity. The Journal of Neuroscience, 36(48), 12276-12292. https://doi.org/10.1523/JNEUROSCI.080916.2016

Fekete, T., Omer, D. B., O'Hashi, K., Grinvald, A., van Leeuwen, C., \& Shriki O. (2018). Critical dynamics, anesthesia and information integration: Lessons from multi-scale criticality analysis of voltage imaging data. Neurolmage, 183, 919-933. https://doi.org/10.1016/j.neuroimage.2018.08.026

Shriki, O., Alstott, J., Carver, F., Holroyd, T., Henson, R. N. A., Smith, M. L., ... Plenz D. (2013). Neuronal avalanches in the resting MEG of the human brain, The Journal of Neuroscience, 33(16), 7079-7090. https://doi.org/10.1523 /JNEUROSCI.4286-12.2013

\section{PLENARY SESSION PRESENTATIONS}

\section{Preventing Murder: Treating Violent Behavior with NFB + BFB}

Lynda Thompson, Michael Thompson

ADD Centre \& Biofeedback Institute of Toronto, Toronto, Ontario, Canada

Violent offenders often present with attentiondeficit/hyperactivity disorder (ADHD). Additionally, violent behavior is associated with frontal lobe damage and, in some cases, with undiagnosed seizure disorders. Neurofeedback has established efficacy for treating both ADHD and epilepsy, so logic supports using neurofeedback in a population prone to committing violent acts. Following that logic, Douglas Quirk treated violent offenders at the Ontario Correctional Institute, eventually publishing some initial findings in 1995. He documented a doseresponse curve: the greater the number of 
neurofeedback plus biofeedback training sessions, the less likely the individual was to reoffend. Documenting that recidivism dropped from $65 \%$ to $20 \%$, he estimated that neurofeedback training prevented more than 100 murders. Quirk died shortly after publication of those findings. This presentation seeks to renew interest in working with this population by reviewing both his work and other research findings that support applying neurofeedback and biofeedback to treat people who display violent behavior. This presentation will review the rationale for applying neurofeedback; namely, that there is frontal lobe dysfunction, including high rates of ADHD, in the population of incarcerated people. This will be followed by a description of the intervention method of neurofeedback combined with appropriate biofeedback. The neurofeedback can be single- or two-channel, but how to assess using 19 channels and treat with LORETA NFB will also be described. The biofeedback modalities are chosen following a stress test that identifies how a client responds to and recovers from mild stressors. The BFB modalities include peripheral skin temperature, skin conduction (electrodermal [EDR]), electromyogram, heart rate, and respiration. There will be an overview of relevant research papers that support major sections of material. The talk will finish with recommendations regarding not only how further applied research might be done in correctional facilities but also with how the neurofeedback practitioner can identify and treat potentially aggressive patients.

\section{References}

Beauregard, M. \& Lévesque, J. (2006). Functional magnetic resonance imaging investigation of the effects of neurofeedback training on the neural bases of selective attention and response inhibition in children with attentiondeficit/hyperactivity disorder. Applied Psychophysiology and Biofeedback, 31(1), 3-20. https://doi.org/10.1007/s10484-0069001-y

Ghaziri, J., Tucholka, A., Larue, V., Blanchette-Sylvestre, M., Reyburn, G., Gilbert, G., Lévesque, J. \& M. Beauregard. (2013). Neurofeedback training induces changes in white and gray matter. Clinical EEG and Neuroscience, 44(4), 265-272. https://doi.org/10.1177/1550059413476031

Nelson, R. J. \& Trainor, B. C. (2007). Neural mechanisms of aggression. Nature Reviews Neuroscience, 8, 536-546. https://doi.org/10.1038/nrn2174

Oded, Y. (2018). Integrating mindfulness and biofeedback in the treatment of posttraumatic stress disorder. Biofeedback, 46(2), 37-47.

Quirk, D. A. (1995). Composite biofeedback donditioning and dangerous offenders: III. Journal of Neurotherapy, 1(2), 44-54. https://doi.org/10.1080/10874208.2012.10491665

Repple, J., Pawliczek, C. M., Voss, B., Siegel, S., Schneider, F. Kohn, N., \& Habel, U. (2017). From provocation to aggression: The neural network. BMC Neuroscience, 18(1), 73. https://doi.org/10.1186/s12868-017-0390-z

Reynolds, R. \& Quirk, D. (2018). A simple and effective cure for criminality. Toronto, Canada: Ex Libris.
Sterman, M. B. (2000). Basic concepts and clinical findings in the treatment of seizure disorders with EEG operant conditioning. Clinical EEG and Neuroscience, 31(1), 45-55. https://doi.org $/ 10.1177 / 155005940003100111$

Takeuchi, H., Taki, Y., Sassa, Y., Hashizume, H., Sekiguchi, A., Fukushima, A., \& Kawashima, R. (2013). Brain structures associated with executive functions during everyday events in a non-clinical sample. Brain Structure and Function, 218(4), 1017-1032. https://doi.org/10.1007/s00429-012-0444-z

Thompson, M., \& Thompson, L. (2015). Functional neuroanatomy: Organized with reference to networks, lobes of the brain, 1020 sites, and Brodmann areas. Wheat Ridge, CO: Association for Applied Psychophysiology.

\section{Infraslow Neurofeedback for Military Trauma Sharie Woelke}

Renew Neurotherapy, Nepean, Ontario, Canada

This workshop will describe infraslow neurofeedback training with soldiers whose symptoms include expressions of posttraumatic stress disorder (PTSD), anxiety, and depression. This didactic presentation will categorize symptoms and identify biological, medical, social, and emotional obstacles. The presenter will review the use of neurofeedback to improve physiological "calming," to follow through with strategy use, and to enhance the effectiveness of interventions.

Infraslow neurofeedback offers a unique opportunity to intervene at the core source of traumatic stress. ISF has been shown to regulate the autonomic nervous system and facilitate behavioral stabilization of related functions (Lecci et al., 2017; Smith, 2013; Smith, Collura, Ferrara, \& de Vries, 2014). ISF neurofeedback's centrality in regulating the excitability cycle of interoceptive networks positively impacts treatment outcomes.

Research suggests that PTSD reflects an abnormal adaptation of neurobiological systems to traumatic stress (DiCara, 1974). This response involves systems that control endocrine pathways and brain networks that regulate, among other functions, the fear response. A primary feature of the PTSD client is persistent hyperactivity of the sympathetic branch of the autonomic nervous system. This system is impacted by the hypothalamic-pituitary-adrenal (HPA) axis: the central coordinator of neuroendocrine stress response (Daniels, Frewen, McKinnon, \& Lanius, 2011; Sherin \& Nemeroff, 2011). Ledoux (LeDoux \& Pine, 2017) has proposed a "two-system" view of fear and anxiety. One system produces behavioral and physiological responses to threat. The other brain circuit produces the conscious feeling-states of fear and anxiety. 
The physiological response to trauma activation is present despite higher level cognitive reasoning. Muscular and physiological reactions are automatic, leaving the conscious capacity to "catch up later" (Van der Kolk, 2014). Activation leads to avoidance, thus increasing the client's disability and negatively impacting quality of life. Although psychotherapy is essential in the recovery process, without the ability to regulate the autonomic nervous system and related limbic networks sufficiently to tolerate the process, participation is often limited or unsuccessful.

By virtue of its ability to support the regulation of the autonomic nervous system/HPA axis (and subsequently the automatic physiological response to trauma), ISF neurofeedback is believed to be a foundational approach to promoting recovery.

\section{References}

Aladjalova, N. A. (1957). Infra-slow rhythmic oscillations of the steady potential of the cerebral cortex. Nature, 179(4567), 957-959. https://doi.org/10.1038/179957a0

Aladjalova, N. A. (1964). Slow electrical processes in the brain (Vol. 7, 1st ed.). Amsterdam: Elsevier Science.

Daniels, J. K., Frewen, P., McKinnon, M. C., \& Lanius, R. A. (2011). Default mode alterations in posttraumatic stress disorder related to early-life trauma: A developmental perspective. Journal of Psychiatry \& Neuroscience, 36(1), 56-59. https://doi.org/10.1503/jpn.100050

DiCara, L. (Ed.). (1974). Limbic and autonomic nervous systems research. New York, NY: Plenum Press.

Lecci, S., Fernandez, L. M. J., Weber, F. D., Cardis, R., Chatton, J.-Y., Born, J., \& Lüthi, A. (2017). Coordinated infraslow neural and cardiac oscillations mark fragility and offline periods in mammalian sleep. Science Advances, 3(2), e1602026. https://doi.org/10.1126/sciadv.1602026

LeDoux, J. E., \& Pine, D. S. (2017). Using neuroscience to help understand fear and anxiety: A two-system framework. The American Journal of Psychiatry, 173(11), 1083-1093. https://doi.org/10.1176/appi.ajp.2016.16030353

Palva, J. M., \& Palva, S. (2012). Infra-slow fluctuations in electrophysiological recordings, bloodoxygenation-leveldependent signals, and psychophysical time series. Neurolmage, 62(4), 2201-2211. https://doi.org/10.1016 /j.neuroimage.2012.02.060

Sherin, J. E., \& Nemeroff, C. B. (2011). Post-traumatic stress disorder: The neurobiological impact of psychological trauma. Dialogues in Clinical Neuroscience, 13(3), 263-278.

Sinha, R. (2008). Chronic stress, drug use, and vulnerability to addiction. Annals of the New York Academy of Sciences, 1141(1), 105-130. https://doi.org/10.1196/annals.1441.030

Smith, M. L. (2013). Infra-slow fluctuation training; On the downlow in neuromodulation. NeuroConnections, Fall 2013, 38-46.

Smith, M. L., Collura, T. F., Ferrara, J., \& de Vries, J. (2014). Infraslow fluctuation training in clinical practice: A technical history. NeuroRegulation, 1(2), 187-207. https://doi.org/10.15540 Inr.1.2.187

Van der Kolk, B. A. (2006). Clinical implications of neuroscience research in PTSD. Annals of the New York Academy of Sciences, 1071(1), 277-293. https://doi.org/10.1196 /annals.1364.022
Van der Kolk, B. A. (2014). The body keeps the score: Brain, mind, and body in the health of trauma. New York, NY: Penguin Books.

Vanhatalo, S., Palva, J. M., Holmes, M. D., Miller, J. W., Voipio, J., \& Kaila, K. (2004). Infraslow oscillations modulate excitability and interictal epileptic activity in the human cortex during sleep. Proceedings of the National Academy of Sciences of the United States of America, 101(14), 5053-5057. https://doi.org/10.1073/pnas.0305375101

\section{Neural Oscillations Induced with Photobiomodulation Could Improve Neurofeedback Outcomes \\ Lew Lim \\ Vielight Inc., Toronto, Ontario, Canada}

Hypothesis. Evidence supports the efficacy of transcranial photobiomodulation (PBM) to affect a wide variety of brain conditions (Hamblin, 2016; Salehpour et al., 2018). It is therefore reasonable to expect that underlying the clinical outcomes, are changes in neural oscillations. Such changes would be measurable with EEG. We could potentially use such data to adjust PBM parameters to modulate the brain to address various brain conditions or even elevate normal brain performance. This would be a novel and useful brain stimulation method for neurofeedback (NFB) practitioners.

Supporting Evidence to Date. In PBM, near infrared (NIR) light directed to the default mode network (DMN) at selected pulses could produce large brain responses. This has been suggested in studies involving dementia (Lim, 2018; Saltmarche, Naeser, Ho, Hamblin, \& Lim, 2017; Zomorrodi, Saltmarche, Loheswaran, Ho, \& Lim, 2017) and acute cognitive processing (Heinrich et al., 2019). In a controlled study that specifically explored neural response to $40 \mathrm{~Hz}$ directed to the DMN of healthy subjects (the Gamma EEG study), it was found that the power spectrum and connectivity of alpha, beta, and gamma increased significantly. Surprisingly, the opposite was found for the slower delta and theta bands where the power spectrum decreased with no significant change in connectivity. In the meantime, there was a global increase in inhibition which is often desirable (Zomorrodi, Loheswaran, Pushparaj, \& Lim, 2019). The changes are observable in qEEG maps, producible with commonly used 19-channel practitioner systems. These information gives a good basis to explore another popular pulse frequency with PBM to determine how much of the effect of the previous study with $40 \mathrm{~Hz}$ can be replicated, and what else can be learned with pulsed PBM. 
Methods. In a new study, we induce a different pulse frequency of $10 \mathrm{~Hz}$. The protocol of this new study is similar to the above study except that a frequency of $10 \mathrm{~Hz}$ is used instead of $40 \mathrm{~Hz}$. It will also be a randomized double-blind study involving 20 healthy participants. In addition, a task stimulus is added to study event-related potential (ERP). Participants cross over to a different device (either from active to sham or vice versa) after a washout period of 2 weeks.

Results. At the time of writing, the study has started but not completed. The results and analysis will focus on the similarity and differences with the Gamma EEG study so that we have new data on the effect of a different set of parameters, plus ERP. It leads to further investigations for personalized treatments.

Conclusions. Although this new study is yet to complete, the data from the Gamma EEG study has already given us considerable bases to make the following conclusions from pulsed PBM: (a) we can produce significant brain modulation that is frequency dependent, (b) it shows the potential for PBM to be an important partner to NFB practice, and (c) it will create clearer opportunities for personalized treatments. Findings in this new study inducing the alpha frequency of $10 \mathrm{~Hz}$ and a task could give us new data to help improve future transcranial PBM treatments.

\section{References}

Hamblin, M. R. (2016). Shining light on the head: Photobiomodulation for brain disorders. BBA Clinical, 6, 113124. https://doi.org/10.1016/j.bbacli.2016.09.002

Heinrich, M. D., Sanguinetti, J. L., Hicks, G., Gibsona, B. C., Mullins, T. S., Aragon, D. F., ... Clark, V. P. (2019, February). Photobiomodulation for cognitive enhancement in healthy adults. Poster presented at the Brain Stimulation Conference 2019, Vancouver, BC, Canada.

Lim, L. (2018). The growing evidence for photobiomodulation as a promising treatment for Alzheimer's disease. Journal of Biosciences and Medicine, 6(12), 100-110. https://doi.org /10.4236/jbm.2018.612010

Salehpour, F., Mahmoudi, J., Kamari, F., Sadigh-Eteghad, S., Rasta, S. H., \& Hamblin, M. R. (2018). Brain photobiomodulation Therapy: A narrative review. Molecular Neurobiology, 55(8), 6601-6636. https://doi.org/10.1007 /s12035-017-0852-4

Saltmarche, A. E., Naeser, M. A., Ho, K. F., Hamblin, M. R., \& Lim, L. (2017). Significant Improvement in Cognition in Mild to Moderately Severe Dementia Cases Treated with Transcranial Plus Intranasal Photobiomodulation: Case Series Report. Photomed Laser Surg. 35(8): 432-441. https://doi.org/10.1089 /pho.2016.4227

Zomorrodi, R., Saltmarche, A. E., Loheswaran, G., Ho, K. F., \& Lim, L. (2017, July). Complementary EEG evidence for a significantly improved Alzheimer's disease case after photobiomodulation treatment. Poster presented at the
Alzheimer's Association International Conference 2017, London, England.

Zomorrodi, R., Loheswaran, G., Pushparaj, A., \& Lim L. (2019). Pulsed near infrared transcranial and intranasal photobiomodulation significantly modulates neural oscillations: A pilot exploratory study. Scientific Reports, 9, 6309. https://doi.org/10.1038/s41598-019-42693-x

\section{The Feasibility and Validity of Cerebellar EEG Biofeed back \\ Robert Thatcher ${ }^{1}$, Ernesto Palmero-Solert ${ }^{2}$, Carl Biver ${ }^{1}$ \\ 'Applied Neuroscience, Inc., Largo, Florida, USA \\ ${ }^{2}$ Université de Lorraine, Nancy, France}

Objectives and Hypotheses. The purpose of this study was to explore the feasibility and validity of cerebellar EEG biofeedback. Cerebellar EEG sources have been published using swLORETA with 128-channel EEG recordings. The questions at hand: Can cerebellar sources also be recorded using only 19 channels, can changes in limb position result in changes in cerebellar sources, and can cerebellar sources be modified using standard EEG biofeedback methods? The null-hypotheses were (a) it is not possible to measure cerebellar current sources using 19-channel EEG, (b) it is not possible to measure cerebellar functional connectivity to other brain regions, (c) it is not possible to change cerebellar EEG sources and/or connectivity by experimental manipulation, and (d) it is not possible to change cerebellar current sources using EEG biofeedback.

Methods. The electroencephalogram (EEG) was recorded from 19 scalp locations referenced to linked ears from 10 normal controls and $10 \mathrm{TBI}$ patients (age 18 to 23 years). The standard MNI-Colin27 MRI with 12,056 voxels was used to compute the inverse solution using swLORETA. The Hilbert transform was used to compute the real-time auto and the cross-spectrum. Current sources from the 10 major cerebellum regions were computed off-line and in real-time (Vermis, lobules IV-VI, VIIB-VIIIB, and flocculus). The measures of functional connectivity were coherence and phase differences between the 10 left hemisphere and the 10 right hemisphere cerebellar center voxels and the center voxels of 44 left hemisphere and 44 right hemisphere Brodmann areas.

Results. All 20 subjects demonstrated cerebellar current sources in all 10 cerebellar regions in both offline analyses and in real-time via playback of the recorded EEG. Similarly, functional and effective connectivity were successfully computed between the cerebellar center voxels and various neocortical 
center voxels, including Brodmann areas 3, 4, 5, and 7 , which are known cerebellar projection areas.

Conclusions. The results of the analyses rejected hypotheses \#1 and \#2 and successfully established the feasibility of measuring cerebellar current sources and functional and effective cerebellar connectivity using 19 EEG channels. Testing of the validity of hypotheses \#3 and \#4 are awaiting the development of the necessary software for left versus right fingerto-nose and point-to-point cerebellar testing and neurofeedback software. We expect to complete the software development and the testing of these two hypotheses around July 2019, in advance of the ISNR conference.

\section{References}

Cebolla, A. M., Petieau, M., Dan, B., Balazs, L., McIntyre, J., \& Cheron, G. (2016). Cerebellar contribution to visuo-attentional alpha rhythm: Insights from weightlessness. Scientific Reports, 6, 37824. https://doi.org/10.1038/srep37824

Cebolla, A.-M., Palmero-Soler, E., Leroy, A. \& Cheron, G. (2017). EEG spectral generators involved in motor imagery: $A$ swLORETA study. Frontiers in Psychology, 8, 2133. https://doi.org/10.3389/fpsyg.2017.02133

Collins, D. L., Neelin, P., Peters, T. M., \& Evans, A. C. (1994). Automatic 3D intersubject registration of MR volumetric data in standardized Talairach space. Journal of Computer Assisted Tomography, 18(2), 192-205. https://doi.org/10.1097 /00004728-199403000-00005

Mazziotta, J. C., Toga, A. W., Evans, A., Fox, P., \& Lancaster, J. (1995). A probabilistic atlas of the human brain: Theory and rationale for its development. The International Consortium for Brain Mapping (ICBM). Neurolmage, 2(2, Part A), 89-101. https://doi.org/10.1006/nimg.1995.1012

Palmero Soler, E., Dolan, K., Hadamschek, V., \& Tass, P.A. (2007). swLORETA: A novel approach to robust source localization and synchronization tomography. Physics in Medicine \& Biology, 52(7), 1783-1800. https://doi.org/10.1088 /0031-9155/52/7/002

Schmahmann, J. D., Doyon, J., McDonald, D., Holmes, C., Lavoie, K., Hurwitz, A. S., ... Petrides, M. (1999). Three-dimensional $\mathrm{MRI}$ atlas of the human cerebellum in proportional stereotaxic space. Neurolmage, 10(3), 233-260. https://doi.org/10.1006 /nimg.1999.0459

\section{Peak Performance in Sports: Factors that Impair Performance and Means of Improving It Jeff Simonson ${ }^{1}$, Dave Siever ${ }^{2}$ \\ ${ }^{1}$ Oregon Tractor, Portland, Oregon, USA \\ ${ }^{2}$ Mind Alive Inc., Edmonton, Alberta, Canada}

Since the development of the Yerkes-Dodson curve of 1908, much has been learned about arousal and its relation to peak performance. Different activities require different levels of arousal for top-notch performance. For instance, the arousal necessary to play a good game of golf is very different than that required for a good game of hockey. Fear from self- criticism, stereotype threat, fear of losing a competition, excitement over the cameras, media, and new adventures often adversely affect performance. Those with the calmest level of disposition often win the competition, even if rated as the underdog prior to competition. However, some athletes start a competition with too low an arousal and do poorly in the beginnings of a competition. Also, many athletes struggle with sleep and often have their worst sleep the night prior to competition.

Audio-visual entrainment (AVE) is a technique employing light and sound stimulation at various frequencies, which in turn can dramatically affect arousal. A peak-performance AVE protocol in combination with the X-ray visualization along with heart rate variability, enhances peak performance and improves sleep, resulting in first-place finishes. Many Olympic and professional athletes have used AVE over the years to gain the mental edge over their opponents. The concepts and techniques surrounding peak performance will be reviewed and demonstrated. Jeff Simonson, a competitive and winning racquetball player will explain how AVE has helped him achieve his first place finishes.

Jeff has been playing tournament racquetball for the past 7 years. Until last year he never finished higher than third place. In 2018 he started using the AVE on a regular basis and specifically a couple hours before a tournament. Last year he started winning tournaments. He's been named to the Oregon All State Racquetball team in 2018 and 2019. Earlier this year he won the Oregon Championships for his division. He is also an avid golfer and has seen his game improve through use of the AVE.

\section{References}

Broadhurst, P. L. (1959). The interaction of task difficulty and motivation: The Yerkes-Dodson law revived. Acta Psychologica, 16, 321-338. https://doi.org/10.1016/00016918(59)90105-2

Hutchison, M. (1993, July). Mind Over Matter = Muscle. Muscle \& Fitness, July, 1-5.

Siever, D., \& Collura, T. (2017). Chapter 3 - Audio-visual entrainment: Physiological mechanisms and clinical outcomes. Rhythmic Stimulation Procedures in Neuromodulation, 51-95. https://doi.org/10.1016/B978-0-12-803726-3.00003-1 
Optical Model of Transcranial Infrared Light and the Penetration Rate to Prefrontal Lobe Li-da Huang ${ }^{1}$, Kung-Bin Sung ${ }^{2}$, Kailin Wang ${ }^{1}$

${ }^{1}$ CytonSys, Lewes, Delaware, USA

${ }^{2}$ National Taiwan University, Taipei City, Taiwan

Brain functions have been proven to be affected by external stimuli. Photobiomodulation (PBM) using near-infrared is one of the effective ways to modulate the hemodynamic activities in the brain and to activate the key enzyme cytochrome c oxidase (CCO) in electron transport chain (ETC; Anders, Lanzafame, \& Arany, 2015; Anders et al., 2014; Barrett \& Gonzalez-Lima, 2013; Blanco, Saucedo, \& GonzalezLima, 2017; Rojas \& Gonzalez-Lima, 2017; Tian, Hase, Gonzalez-Lima, \& Liu, 2016; Vargas et al., 2017; Wang, Tian, Soni, Gonzalez-Lima, \& Liu, 2016). However, the effective dosage, or the right recipe for the power density as well as treatment time, is still not clear. To answer these questions, it is necessary to have a detailed optical model to describe the penetration rate and how photons propagate transcranially, particularly to reach the prefrontal lobe that dominates the cognitive functions. In this research, we used five layers of tissues including skin, skull, cerebrospinal fluid (CSF), gray matter, and white matter, each of which is represented by a set of wavelength-dependent Scattering coefficients $\mu s \quad(\lambda)$ and Absorption coefficients $\mu \mathrm{a}(\lambda)$. The anisotropy factor $g$ is needed to run Monte Carlo simulations and predict the reflectance (fraction of light returning to tissue surface; Sung, Kao, Zhan, \& Lin, 2019). A broadband near-infrared light source emits photons through the prefrontal area, and a linear array with 10 sensors spacing $10 \mathrm{~mm}$ from each other collects the photons scattering back from the superficial and deep tissues. Based on these collected data, the Monte Carlo model is built, and simulations are executed in highspeed parallel computers. The thickness of each layer of the model is from the MRI result of participants. Through the Monte Carlo simulation results, we identified that penetration rate for the skull is about $3.5 \%$ and the photons could reach as deep as $4 \mathrm{~cm}$ to the grey matter, with about $2 \%$ of photons. In addition, we also noticed that photons have the longest path distance horizontally in the skull layer. This indicates that photons travel longer than expected as in previous studies. Therefore, the shortchannel effect completely cannot be ignored when using near-infrared spectroscopy (NIRS) techniques (Wang et al., 2016) to measure the activities of the $\mathrm{CCO}$ at the prefrontal lobe after the PBM treatment.

\section{References}

Anders, J. J., Lanzafame, R. J., \& Arany, P. R. (2015). Low-level light/laser therapy versus photobiomodulation therapy. Photomedicine and Laser Surgery, 33(4), 183-184. https://doi.org/10.1089/pho.2015.9848

Anders, J. J., Moges, H., Wu, X., Erbele, I. D., Alberico, S. L., Saidu, E. K., ... Pryor, B. A. (2014). In vitro and in vivo optimization of infrared laser treatment for injured peripheral nerves. Lasers in Surgery and Medicine, 46(1), 34-45. https://doi.org /10.1002/lsm.22212

Barrett, D. W., \& Gonzalez-Lima, F. (2013). Transcranial infrared laser stimulation produces beneficial cognitive and emotional effects in humans. Neuroscience, 230, 13-23. https://doi.org /10.1016/j.neuroscience.2012.11.016

Blanco, N. J., Saucedo, C. L., \& Gonzalez-Lima, F. (2017). Transcranial infrared laser stimulation improves rule-based, but not information-integration, category learning in humans. Neurobiology of Learning and Memory, 139, 69-75. https://doi.org/10.1016/j.nlm.2016.12.016

Rojas, J. C., \& Gonzalez-Lima, F. (2017). Transcranial low-level laser (light) therapy for neurocognitive enhancement. In M. R. Hamblin, M. V. P. de Sousa, \& T. Agrawal (Eds.), Handbook of low-level laser therapy (1st ed., pp. 1057-1076). Singapore: Pan Stanford Publishing.

Sung, K.-B., Kao, T.-C., Zhan, C.-S., \& Lin, T.-X. (2019, April). Comparison of photon energy distributions in the prefrontal cortex between $810 \mathrm{~nm}$ and $1064 \mathrm{~nm}$ for optimizing photobiomodulation effects. Paper presented at the Biophotonics Congress: Optics in the Life Sciences Congress 2019 (BODA, BRAIN, NTM, OMA, OMP), The Optical Society (Optical Society of America, 2019), Tucson, AZ https://doi.org/10.1364/BRAIN.2019.BW4A.5

Tian, F., Hase, S. N., Gonzalez-Lima, F., \& Liu, H. (2016). Transcranial laser stimulation improves human cerebral oxygenation. Lasers in Surgery and Medicine, 48(4), 343-349. https://doi.org/10.1002/lsm.22471

Vargas, E., Barrett, D. W., Saucedo, C. L., Huang, L.-D., Abraham, J. A., Tanaka, H., ... Gonzalez-Lima, F. (2017). Beneficial neurocognitive effects of transcranial laser in older adults. Lasers in Medical Science, 32(5), 1153-1162. https://doi.org /10.1007/s10103-017-2221-y

Wang, X., Tian, F., Soni, S. S., Gonzalez-Lima, F., \& Liu, H. (2016). Interplay between up-regulation of cytochrome-c-oxidase and hemoglobin oxygenation induced by near-infrared laser Scientific Reports, 6, 30540. https://doi.org/10.1038 /srep30540

\section{All Artifacts \\ Tiff Thompson \\ NeuroField Neurotherapy, Inc., Santa Barbara, California, USA}

This course is for anyone who has wondered: "Is that real or fake?" Artifacts in EEG can seriously hinder an accurate interpretation of the qEEG, altering neurotherapeutic protocol choices and potentially threatening the patient's clinical outcome. Muscle tension can look like anxiety, lateral eye movements and pulse artifacts can create delta and the misinterpretation of head injury, or a contaminated ear electrode can create hypercoherence! This presentation will demonstrate how artifacts appear not only in the raw EEG data, but also in the qEEG 
data. Tiff hopes to spare you and your patients this dilemma in the future, as well as provide tips on how to avoid any misread of quantitative and raw EEG data.

While some artifact can be prevented or omitted, other artifact must be read around. The skilled clinician needs the ability to explain these findings to their patients when asked, "what's that?" Through exploring the more and less insidious forms of artifact, this lecture will guide the clinician through some of the more advanced ways of interpreting EEG so that the qEEG does not mislead one into misdiagnosis.

Some of the artifacts covered will include channel noise, $60 \mathrm{~Hz} / 50 \mathrm{~Hz}$, blinks, chewing, EKG (various presentations), electrode pops, eye rolling, sweat, lateral eye movements, medication, mixed metals, muscle tension, shared variance, as well as easily confused real conditions.

This presentation will be simple enough for the novice neurotherapist but rich enough that even the most seasoned clinician should be able to glean something new.

\section{References}

Johnstone, J. (2005, September). QEEG patterns: Subgroups, profiles, phenotypes. Paper presented at the 13th Annual Conference of the International Society for Neuronal Regulation (ISNR), Denver, CO.

La Vaque, T. J., Hammond, D. C., Trudeau, D., Monastra, V., Perry, J., Lehrer, P., ... Sherman, R. (2002). Template for developing guidelines for the evaluation of the clinical efficacy of psychophysiological interventions. Applied Psychophysiology and Biofeedback, 27(4), 273-281. https://doi.org/10.1023/A:1021061318355

Niedermeyer, E., \& Lopes da Silva, F. (1993). EEG patterns and genetics. In E. Niedermeyer \& F. Lopes da Silva, Electroencephalography: Basic principles, clinical applications and related fields (3rd ed., pp. 192-195). Baltimore, MD: Lippincott, Williams \& Wilkins.

Prichep, L. S., \& John, E. R. (1992). QEEG profiles of psychiatric disorders. Brain Topography, 4(4), 249-257. https://doi.org /10.1007/BF01135562
Correlations Between Quantitative EEG

Volumetric Analysis and Computerized

Cognitive Testing Shortly After Sport

Concussion Injury in High School Athletes

Harry Kerasidis, Paul David Ims, Stacie Rector, Stephanie Guzzo, Andrew Ims, Annastassia Kerasidis

Chesapeake Neurology Associates, Prince Frederick, Maryland, USA

The objective of this investigation is to explore the relationship between symptoms and cognitive performance and volumetric qEEG analysis after sport concussion injury in high school athletes.

Methods. Standard electroencephalograms (EEGs) were recorded in 70 high school athletes ( 31 males) shortly after concussion injury using sLORETA imaging compared to a normative database (NYU/BrainDx). Peak Z-score variation (PZV), and percentage volume of grey matter activity that fell outside $Z=-2.5$ to 2.5 (PIGMV for increased activity, PRGMV for reduced) were calculated for each of five EEG frequency bands. These data were compared for correlations to computerized neurocognitive and symptom assessment (XLNTbrain) also performed shortly after concussion injury.

Results. Statistically significant Pearson $r$ correlations were found with XLNTbrain composite scores. For PZV: Negative correlations between delta band PZV min values and nonverbal processing and memory scores $(r=-.334, p=.005 ; r=-.339, p$ $=.004$; respectively). Positive correlations were found with alpha band PZV max value and verbal memory score $(r=.319, p=.007)$. For PIGMV: Positive correlations were found between alpha band PIGMV and verbal processing, verbal memory, attention, and emotional reactivity $(r=.341, p=.004$; $r=.351, p=.003 ; r=.244, p=.042 ; r=.254, p=.034$; respectively).

Taking reaction time into account with throughput measures, for PZV: Negative correlation was found between delta band PZV min and nonverbal memory. Positive correlations were found between alpha band PZV max and verbal memory. For PIGMV: Positive correlations were found between alpha band PIGMV and verbal processing and verbal memory $(r=.28,=$ $.019 ; r=.314, p=.008$; respectively) and the betagamma band and nonverbal processing and nonverbal memory $(r=.28, p=.019 ; r=.3, p=.012$; respectively).

Conclusions. This study demonstrates correlations between performance on computerized neurocognitive tasks and changes in quantitative 
sLORETA EEG analysis shortly after concussion injury in high school athletes. The data suggest that different cognitive processes may be supported by different frequency band activity. Further research including region specific EEG analysis and gender differences is needed.

\section{References}

Barr, W. B., Prichep, L. S., Chabot, R., Powell, M. R., \& McCrea, M., (2012). Measuring brain electrical activity to track recovery from sport-related concussion. Brain Injury, 26(1), 58-66. https://doi.org/10.3109/02699052.2011.608216

Bricolo, A., Turazzi, S., Faccioli, F., Odorizzi, F., Sciaretta, G., \& Erculiani, P. (1978). Clinical application of compressed spectral array in long-term EEG monitoring of comatose patients. Electroencephalography and Clinical Neurophysiology, 45(2), 211-225. https://doi.org/10.1016 10013-4694(78)90005-6

Cannon, R., Baldwin, D. R., Shaw, T. L., Diloreto, D. J., Phillips, S. M., Scruggs, A. M., \& Riehl, T. C. (2012) Reliability of quantitative EEG (qEEG) measures and LORETA current source density at 30 days. Neuroscience Letters, 518(1), 2731. https://doi.org/10.1016/j.neulet.2012.04.035

Cannon, R., Kerson, C., \& Hampshire, A. (2011). sLORETA and fMRI detection of medial prefrontal default network anomalies in adult ADHD. Journal of Neurotherapy, 15(4), 358-373, https://doi.org/10.1080/10874208.2011.623093

Duffy, F. H., Bartels, P. H., \& Burchfiel, J. L. (1981). Significance probability mapping: An aid in the topographic analysis of brain electrical activity. Electroencephalography and Clinical Neurophysiology, 51(5), 455-462. https://doi.org/10.1016 /0013-4694(81)90221-2

Duncan, C. C., Kosmidis, M. H., \& Mirsky, A. F. (2003). Eventrelated potential assessment of information processing after closed head injury. Psychophysiology, 40(1), 45-59. https://doi.org/10.1111/1469-8986.00006

Duncan, C. C., Kosmidis, M. H., \& Mirsky, A. F. (2005). Closed head injury-related information processing deficits: An eventrelated potential analysis. International Journal of Psychophysiology, 58(2-3), 133-157. https://doi.org/10.1016 /j.ijpsycho.2005.05.011

Giza, C. C., \& Hovda, D. A. (2014). The new neurometabolic cascade of concussion. Neurosurgery, 75(Suppl. 4), S24-S33. https://doi.org/10.1227/NEU.0000000000000505

Gosselin, N., Bottari, C., Chen, J.-K., Huntgeburth, S. C., De Beaumont, L., Petrides, M., ... Ptito, A. (2012). Evaluating the cognitive consequences of mild traumatic brain injury and concussion by using electrophysiology. Neurosurgical Focus, 33(6), E7. https://doi.org/10.3171/2012.10.FOCUS12253

Gosselin, N., Lassonde, M., Petit, D., Leclerc, S., Mongrain, V., Collie, A., \& Montplaisir, J. (2009). Sleep following sportrelated concussions. Sleep Medicine, 10(1), 35-46. https://doi.org/10.1016/j.sleep.2007.11.023

Haglund, Y., \& Persson, H. E. (1990). Does Swedish amateur boxing lead to chronic brain damage. 3.A retrospective clinical neurophysiological study. Acta Neurologica Scandinavica, 82(6), $\quad 353-360 . \quad$ https://doi.org/10.1111/j.16000404.1990.tb03316.x

Hanley, D., Prichep, L. S., Bazarian, J., Huff, J. S., Naunheim, R., Garrett, J., ... Hack, D. C. (2017) Emergency department triage of traumatic head injury using a brain electrical activity biomarker: A multisite prospective observational validation trial. Academic Emergency Medicine, 24(5), 617-627. https://doi.org/10.1111/acem.13175
Jasper, H. H. (1958). The ten-twenty electrode system of the International Federation. Electroencephalography and Clinical Neurophysiology, 10, 371-375.

John, E. R. (1977). Neurometrics: Clinical applications of quantitative electrophysiology. Hillsdale, NJ: L. Erlbaum Associates.

Koberda, L. (2015). Z-Score LORETA neurofeedback as a potential therapy in cognitive dysfunction and dementia. Journal of Psychology \& Clinical Psychiatry, 1(6), 00037. https://doi.org/10.15406/jpcpy.2014.01.00037

Kutas, M., \& Hillyard, S. A. (1980). Reading senseless sentences: Brain potentials reflect semantic incongruity. Science, 207(4427), 203-205. https://doi.org/10.1126/science.7350657

Ledwidge, P. S., \& Molfese, D. L. (2016). Long-term effects of concussion on electrophysiological indices of attention in varsity college athletes: An event-related potential and standardized low-resolution brain electromagnetic tomography approach. Journal of Neurotrauma, 33(23), 2081-2090. https://doi.org/10.1089/neu.2015.4251

McCrea, M., Prichep, L., Powell, M., Chabot, R., \& Barr, W. (2010). Acute effects and recovery after sport-related concussion: $A$ neurocognitive and quantitative brain electrical activity study. Journal of Head Trauma Rehabilitation, 25(4), 283-292. https://doi.org/10.1097/HTR.0b013e3181e67923

NIH SBIR Contract \# NS72370-000, Rest Technologies, Chris Mann, Jerald Simmons, Continuous EEG Monitoring Methodologies, Awarded 1997.

Pascual-Marqui, R. D. (1999). Review of methods for solving the EEG inverse problem. International Journal of Bioelectromagnetism, 1(1), 75-86.

Pascual-Marqui, R. D. (2002). Standardized low-resolution brain electromagnetic tomography (sLORETA): Technical details. (2002) Methods \& Findings in Experimental \& Clinical Pharmacology, 24(Suppl. D), 5-12.

Schoenhuber, R., Gentilini, M., \& Orlando, A. (1988). Prognostic value of auditory brain-stem responses for late postconcussion symptoms following minor head injury. Journal of Neurosurgery, 68(5), 742-744. https://doi.org/10.3171 /jns.1988.68.5.0742

Thatcher, R. W., Walker, R. A., Biver, C. J., North, D. N., \& Curtin, R. (2003). Quantitative EEG normative databases: Validation and clinical correlation. Journal of Neurotherapy, 7(3-4), 87121. https://doi.org/10.1300/J184v07n03_05

Thatcher, R. W., Walker, R. A., Gerson, I., Geisler, F. H. (1989). EEG discriminant analyses of mild head trauma, Electroencephalography and Clinical Neurophysiology, 73(2), 94-106. https://doi.org/10.1016/0013-4694(89)90188-0

Thompson, M., Thompson, L., \& Reid-Chung, A. (2015). Treating postconcussion syndrome with LORETA Z-Score neurofeedback and heart rate variability biofeedback: Neuroanatomical/neurophysiological rationale, methods, and case examples. Biofeedback, 43(1), 15-26. https://doi.org /10.5298/1081-5937-43.1.07

Vespa, P. M., Nuwer, M. R., Juhász, C., Alexander, M., Nenov, V., Martin, N., \& Becker, D. P. (1997). Early detection of vasospasm after acute subarachnoid hemorrhage using continuous EEG ICU monitoring. Electroencephalography and Clinical Neurophysiology, 103(6), 607-615. https://doi.org/10.1016/s0013-4694(97)00071-0

Vitacco, D., Brandeis, D., Pascual-Marqui, R., \& Martin, E. (2002). Correspondence of event-related potential tomography and functional magnetic resonance imaging during language processing. Human Brain Mapping, 17(1), 4-12. https://doi.org /10.1002/hbm. 10038 
Integration of Neurofeedback in the Drug Court Treatment Model: A Pilot Study

David Ims, Harry Kerasidis

Chesapeake Neurology Associates, Prince Frederick, Maryland, USA

Introduction. In their landmark 1989 paper, Peniston and Kulkosky used alpha-theta neurofeedback to promote alpha and theta wave production in alcoholics and improve treatment results. The EEG profiles of alcoholics often demonstrated reductions in alpha activity and alpha reactively increased in these individuals when alcohol was consumed (Pollack et al., 1983). Results from Peniston and Kulkosky's original methodology indicated increased alpha and theta brainwave production, normalized personality measures, and prolonged prevention of relapse for the neurofeedback group (Peniston, 1998). Research has suggested that the application of alpha-theta neurofeedback is beneficial in promoting long-term abstinence (Fisher, 2009). Independent studies with minor variations to the neurofeedback protocol have achieved similar success (Scott, Kaiser, Othmer, \& Sideroff, 2005). Since 1989, drug court programs have integrated the leverage of the criminal justice system to addiction treatment services (Ritvo, Martin, \& Fehling, 2015). The current study sought to apply neurofeedback within the context of a drug court program. The study participants were female members of an internal family systems (IFS) therapy group which had met for 16 weeks as required by drug court.

Methods. Pretreatment baseline and posttreatment qEEG data, cognitive testing data, and subjective symptom scores were acquired before and after treatment. Twice weekly, single-channel electrode neurofeedback training was performed on a BrainMaster Atlantis amplifier in BrainMaster $3.7 \mathrm{i}$ software. Twenty sessions were planned but 19 were performed due to inclement weather. Each client first completed five sessions of beta-type training. Alphatheta training was applied during the remaining sessions.

Results. The subjective experiences of all three study participants improved with the neurofeedback sessions. QEEG analysis used $\mathrm{ANI}$ and BrainDx databases. Each participant in the study demonstrated changes in qEEG profiles over the course treatment. Alpha and beta bands demonstrated the most remarkable changes. Symptom scores reduced and trended towards improvement in two of three cases, while the last case remained elevated at a near constant level. Cognitive testing measures were varied and unreliable due to testing error.

Discussion. While changes in the objective measures was mixed, making it difficult to draw concrete conclusions from this case series, dramatic changes were noted on some qEEG maps. No subjects relapsed during the neurofeedback program. In follow-up discussions all subjects reported improved self-regulation, emotional regulation, and executive functioning. Group therapy facilitators and drug court council personnel noted changes in the participants' demeanor, mood, and personality. Follow-up with the drug court council 2 months later revealed all three study participants were doing well and were on track to graduate from the drug court program within 3 months.

Conclusion. Study limitations and variance of objective results in the present study prevent clear conclusions about the efficacy of neurofeedback in this model, but interviews with the study participants, group therapy facilitators, and drug court council suggest neurofeedback had a positive effect. Future research is needed and currently a second study to use neurofeedback with individuals recovering in the drug court program is in development.

\section{References}

Fisher, C. (2009). An overview of alpha-theta neurofeedback and its treatment effectiveness for substance abuse. Retrieved from http://www.bmedreport.com/archives/6071

Peniston, E. G. (1998). The Peniston-Kulkosky brainwave neurofeedback therapeutic protocol: The future of psychotherapy for alcoholism/PTSD/behavioral medicine. Retrieved from http://www.aaets.org/article47.htm

Peniston, E. G. \& Kulkosky, P. J. (1989). Alpha-theta brainwave training and beta-endorphin levels in alcoholics. Alcoholism: Clinical and Experimental Research, 13(2), 271-279. https://doi.org/10.1111/j.1530-0277.1989.tb00325.x

Pollack, V. E., Volavka, J., Goodwin, D. W., Medrick, S. A., Gabrielli, W. F., Knop, J., \& Schulsinger, F. (1983). The EEG after alcohol in men at risk for alcoholism. Archives of General Psychiatry, 40(8), 857-864.

Ritvo, J. I., Martin, L. F., \& Fehling, P. D. (2015). Community-based treatment. In M. Galanter, H. D. Kleber, \& K. Brady (Eds.), The American Psychiatric Publishing Textbook of Substance Abuse Treatment (5th ed., pp. 531-543). Washington, DC: American Psychiatric Publishing.

Scott, W. C., Kaiser, D., Othmer, S., \& Sideroff, S. I. (2005). Effects of an EEG biofeedback protocol on a mixed substance abusing population. The American Journal of Drug and Alcohol Abuse, 31(3), 455-469. https://doi.org/10.1081/ada-200056807 
Heart Rate Variability: Psychological Factors and a Novel Technique for Dramatically

\section{Enhanced Results}

Dave Siever ${ }^{1}$, John LeMay²

${ }^{1}$ Mind Alive Inc., Edmonton, Alberta, Canada

${ }^{2}$ Saybrook University, Pasadena, California, USA

Heart rate variability (HRV) is often referred to as a "window to the soul," a reflection of thoughts and emotions within the individual in which the heart is contained. And yet, for most heart rate variability biofeedback, a simplistic approach has been developed, practiced, and-unfortunately-accepted as status quo for the treatment of HRV issues.

$\mathrm{HRV}$ issues are prevalent in relation to traumatic brain injury and mental health conditions in which anxiety is a major presenting symptom. Also, it appears that the more anxious or afraid of failing that one is, the more likely that person will fail when attempting to breathe to a regimented fashion. So, a regimented and paced biofeedback system is particularly distressing to these sufferers. Suppose there was a technique that simultaneously calmed the mind while also providing gentle breathing cues?

The essence of this workshop will be to bring light the way in which the heart's rhythm is of absolute importance as a guide for counselling and cognitive behavioral techniques, which in themselves can completely resolve an HRV issue. That being said, the more extreme a mental health condition is, especially that of PTSD, trauma, TBI and the resultant anxiety, the tougher it is to employ traditional HRV biofeedback techniques and the greater the failure rate.

Audio-visual entrainment (AVE) is a technique employing flashing lights within a pair of sunglasses and pulsing tones within a pair of headphones at various brain wave frequencies. AVE has the exceptional ability to induce deep meditative and parasympathetic states of mind within the user. This workshop will demonstrate a novel and highly effective method involving an AVE-based technique involving a Windows-based Breathe app alongside a unique AVE protocol for advanced HRV therapy for the toughest of patients struggling with anxiety. This protocol utilizes the changing of color cues for inspiration and expiration, simultaneously with mindcalming AVE, typically in the alpha brain wave band (although any brain wave band may be chosen).

\section{References}

Moss, D. (2004). Heart rate variability and biofeedback. Psychophysiology Today: The Magazine for Mind-Body Medicine, 1, 4-11.

Siever, D. (2012). Heart rate variability and Audio-visual entrainment. Retrieved from http://mindalive.com/index.cfm /research/hrv-ave/

Winkelmann, T., Thayer, J. F., Pohlack, S., Nees, F., Grimm, O., \& Flor, H. (2017). Structural brain correlates of heart rate variability in a healthy young adult population. Brain Structure and Function, 222(2), 1061-1068. https://doi.org/10.1007 /s00429-016-1185-1

Understanding the Neurophysiology of PTSD, Moral Injury, and Posttraumatic Growth: How

It Can Guide Training Protocols

Manuel Halter

United States Special Operations Command (USSOCOM),

Tampa, Florida, USA

Posttraumatic stress is distinct from moral injury. The former is a natural, automatic response to defend against danger, whereas the latter is the interpretation of such an event, in light of an individual's values, beliefs, and world view. Moral injury, unlike posttraumatic stress, occurs when a person is able to reflect upon a traumatic experience after the immediate danger has passed (i.e., interpretation; Ames et al., 2019). PTSD is a fear reaction to danger and has identifiable trauma symptoms such as flashbacks, nightmares, hypervigilance, and dissociation. On the other hand, moral injury is an inner conflict based on a moral evaluation of having inflicted harm, a judgment grounded in a sense of personal agency. Moral injury is strongly related to negative consequences associated with war-zone stressors that transgress military veterans' deeply held values and beliefs (Currier, Drescher, Holland, Lisman, \& Foy, 2016). Currier, Holland, and Malott (2015) hypothesized that lack of meaning increases the risk for adjustment problems after war-zone service. Moral injury describes the effects of acts of commission or omission in war that result in mental, emotional, and spiritual struggle (Currier et al., 2016). In the past 15 years, the literature on disasters and mental health has shifted from a focus on psychopathology, to an interest in documenting manifestations of resilience in the face of mass trauma (Cerdá, 2014). Positive psychology has provided a new forum for discussion about how we construe mental health issues. Each generation and each culture face basic questions about the meaning of birth, suffering, and dying. Each has its own social constructions and ways of managing these very basic human experiences (Joseph, 2009). Posttraumatic growth is the positive 
psychological change experienced as a result of a struggle with challenging life circumstances that represent significant challenges to the adaptive resources of the individual and an individual's way of understanding the world and his place in it. This positive change depends on an individual's perceived control that he has over a stressor, which in turn determines the stressor's impact. From a neurophysiological perspective, and individual's perceived control over a stressful situation suggests that the dorsal raphe nucleus (DRN) in the brainstem and the ventral medial prefrontal cortex (mPFCv; i.e., Brodmann areas 24, 25, and 32) are key in this process. Indeed, the prelimbic and infralimbic areas of the mPFCv ascertain whether a stress inducing stimulus is under an individual's control. Therefore, when an individual perceives that such a stimulus is under his control, the stress-induced activation of the DRN is mediated or inhibited by the mPFCV, and the effects of the "uncontrollable" stressor are thereby blocked. This process implies that the perceived manifestation of control mediates the stress response that is triggered in the brainstem. Thus, as a practical matter the negative impact of stressors that cause dysregulation of the autonomic nervous system can be mitigated via entraining the functional neural networks that involve the mPFCv and DRN via biofeedback and neurofeedback protocols.

\section{References}

Ames, D., Erickson, Z., Youssef, N. A., Arnold, I., Adamson, C. S., Sones, A. C., ... Koenig, H. G. (2019). Moral injury, religiosity, and suicide risk in U.S. veterans and active duty military with PTSD symptoms. Military Medicine, 184(3-4), e271-e278. https://doi.org/10.1093/milmed/usy148

Cerdá, M. (2014). Posttraumatic growth in the aftermath of a disaster: Looking for the role of gender. Social Psychiatry and Psychiatric Epidemiology, 49(12), 1859-1860. https://doi.org /10.1007/s00127-014-0949-2

Currier, J. M., Drescher, K. D., Holland, J. M., Lisman, R., \& Foy, D. W. (2016). Spirituality, forgiveness, and quality of life: Testing a mediational model with military veterans with PTSD. The International Journal for the Psychology of Religion, 26(2), 167-179. https://doi.org/10.1080/10508619.2015.1019793

Currier, J. M., Holland, J. M., \& Malott, J. (2015). Moral injury, meaning making, and mental health in returning veterans. Journal of Clinical Psychology, 71(3), 229-240. https://doi.org /10.1002/jclp.22134

Joseph, S. (2009). Growth following adversity: Positive psychological perspectives on posttraumatic stress. Psihologijske Teme [Psychological Topics], 18(2), 335-343.
Emotions: Exposing the Neurological Pathways that Far Too Often Control Our Behaviors

Ronald Bonnstetter ${ }^{1}$, Thomas Collura ${ }^{2}$

${ }^{1}$ Target Training International, Scottsdale, Arizona, USA

${ }^{2}$ BrainMaster Technologies, Inc., Bedford, Ohio, USA

Social science literature offers many models of human emotions. Each model provides descriptive terminology but for the most part fails to identify the neuronetworks that are activated when elicited by external emotionally laden stimuli. Historically, science has viewed emotions as primitive and instinctive responses that were not associated with complex intellectual or cognitive functions. However, cognitive-emotional interactions are extremely important in everyday decision-making and represent a significant area of study at present. In addition, the brain has a striking capacity to learn and remember the emotional significance of diverse stimuli and memorial events. Furthermore, the thought process allows humans to assign emotional valence to stimuli, and as a result can change the value that was previously assigned to a stimulus. Studies of brain functions reveal that neural pathways exist for these important cognitive-emotional interactions, but crosswalking these cognitive-emotional interactions to widely used behavioral assessments is missing from the literature. Electroencephalographic (EEG) electromagnetic tomographic analysis (ETA) imaging techniques provide a mechanism for exposing neurological pathways of the emotional spectrum, while an individual is reliving emotional loaded past experiences. This presentation will focus on frontal EEG gamma band asymmetry, at the precognitive level and then will expand this examination to include other frequencies as well as other key subcortical structures.

During the presentation, a number of experimental findings will be shared, including brain imaging of emotional triggers using the categories of fear, joy, anger, and love. Experimental protocols require that each participant complete a behavioral insights assessment survey prior to coming to the lab for an EEG session. Participants are asked to write down three to five stimuli (single words or short phrases) for each of the four categories. The stimuli represent events that they believe will invoke feelings that fall into one of these categories. Next, they are asked to rate how strong they believe the emotion response will be on a Likert scale. While connected for EEG data collection, each stimulus is presented on screen for a period of $2 \mathrm{~s}$ and spaced with a blank screen with a randomized time between 3 to 5 seconds before a new stimulus is presented. The EEG process is 
followed by a postprocess interview. Participants are asked to review each of the stimuli they provided and reflect on their experience. The debrief includes asking if any particular stimuli stood out to them as being stronger or weaker than they originally thought, as well as exploring if any stimuli seemed to provoke additional thought or reflection when presented. All feedback is recorded and cross-referenced to EEG output as well as a comparison to their behavioral style as identified in their behavioral assessment profile.

Documenting the pathways exposed for each of these four emotional categories and then cross-referencing findings to a populate behavioral assessment is intended to provide new insights into the emotional tendencies of various behavioral styles and expose how different behavioral styles may process emotional stimuli differently.

\section{References}

Bonnstetter, B. J., Bonnstetter, R. J., Hebets, D., \& Collura, T. F. (2015). U.S. Patent No. 9,060,702. Washington, DC: U.S. Patent and Trademark Office.

Bonnstetter, B. J., Bonnstetter, R. J., Hebets, D., \& Collura, T. F. (2016). Canadian Patent No. 2,808,691. Gatineau, Québec: Canadian Intellectual Property Office.

Bonnstetter, R. J., Gehrig, E., \& Hebets, D. (2018). Response process validation protocol using neurophenomenological gamma asymmetry. NeuroRegulation, 5(3), 93-102. https://doi.org/10.15540/nr.5.3.93

Bonnstetter, R. J., Hebets, D. \& Wigton, N. L. (2015). Frontal gamma asymmetry in response to soft skills stimuli: A pilot study. NeuroRegulation, 2(2), 70-85. https://doi.org/10.15540 /nr.2.2.70

Collura, T. F., Wigton, N. L., Zalaquett, C., Chatters-Smith, S. S., \& Bonnstetter, R. J. (2016). The value of EEG-based electromagnetic tomographic analysis in human performance and mental health. Biofeedback, 44(2), 58-65. https://doi.org /10.5298/1081-5937-44.2.03

Collura, T. F., Zalaquett, C. P., Bonnstetter, R. J., \& Chatters, S. J. (2014). Toward an operational model of decision making, emotional regulation, and mental health impact. Advances in Mind-Body Medicine, 28(4), 18-33.

Damasio, A. R., Tranel, D. \& Damasio, H. (1991). Somatic markers and the guidance of behaviour: Theory and preliminary testing. In H. S. Levin, H. M. Eisenberg \& A. L. Benton (Eds.), Frontal lobe function and dysfunction (pp. 217-229). New York, NY: Oxford University Press.

Davidson, R. J. (1992). Emotion and affective style: Hemispheric substrates. Psychological Science, 3(1), 39-43. https://doi.org /10.1111/j.1467-9280.1992.tb00254.x

Davidson, R. J. (2000). Affective style, psychopathology, and resilience: Brain mechanisms and plasticity. American Psychologist, 55(11), 1196-1214. https://doi.org/10.1037 /0003-066X.55.11.1196

Davidson, R. J. (2002). Anxiety and affective style: Role of prefrontal cortex and amygdala. Biological Psychiatry, 51(1), 68-80. https://doi.org/10.1016/S0006-3223(01)01328-2

Davidson, R. J. (2004). What does the prefrontal cortex "do" in affect: Perspectives on frontal EEG asymmetry research. Biological Psychology, 67(1-2), 219-234. https://doi.org /10.1016/j.biopsycho.2004.03.008
Davidson, R. J., Ekman, P., Saron, C. D., Senulis, J. A., \& Friesen, W. V. (1990). Approach-withdrawal and cerebral asymmetry: Emotional expression and brain physiology: I. Journal of Personality and Social Psychology, 58(2), 330-341. https://doi.org/10.1037/0022-3514.58.2.330

Davidson, R. J., Pizzagalli, D., Nitschke, J. B., \& Kalin, N. H. (2003). Parsing the subcomponents of emotion and disorders of emotion: Perspectives from affective neuroscience. In R. J. Davidson, K. R. Scherer, \& H. H. Goldsmith (Eds.), Series in affective science. Handbook of affective sciences (pp. 8-24). New York, NY: Oxford University Press.

Davidson, R. J., Schwartz, G. E., Saron, C., Bennett, J., \& Goleman, D. J. (1979). Frontal versus parietal EEG asymmetry during positive and negative affect. Psychophysiology, 16, 202-203.

Gordon, E., Barnett, K. J., Cooper, N. J., Tran, N., \& Williams, L. M. (2008). An "integrative neuroscience" platform: Application to profiles of negativity and positivity bias. Journal of Integrative Neuroscience, 7(3), 345-366. https://doi.org/10.1142 /S0219635208001927

Haidt, J. (2001). The emotional dog and its rational tail: A social intuitionist approach to moral judgment. Psychological Review. 108(4), 814-834. https://doi.org/10.1037/0033-295x.108.4.814

Harmon-Jones, E. (2004). Contributions from research on anger and cognitive dissonance to understanding the motivational functions of asymmetrical frontal brain activity. Biological Psychology, 67(1-2), 51-76. https://doi.org/10.1016 /j.biopsycho.2004.03.003

Kim, J. (1993). Supervenience and mind: Selected philosophical essays. Cambridge, UK: Cambridge University Press.

Kim, J. (1999). Making sense of emergence. Philosophical Studies, 95(1-2), 3-36. https://doi.org/10.1023/A:1004563122154

Nitschke, J. B., Heller, W., Etienne, M. A., \& Miller, G. A. (2004). Prefrontal cortex activity differentiates processes affecting memory in depression. Biological Psychology, 67(1-2), 125143. https://doi.org/10.1016/j.biopsycho.2004.03.004

Naccache, L., Gaillard, R., Adam, C., Hasboun, D., Clémenceau, S., Baulac, M., ... Cohen, L. (2005). A direct intracranial record of emotions evoked by subliminal words. Proceedings of the National Academy of Sciences of the United States of America, 102(21), 7713-7717. https://doi.org/10.1073 /pnas.0500542102

Oakes, T. R., Pizzagalli, D. A., Hendrick, A. M., Horras, K. A., Larson, C. L., Abercrombie, H. C., ... Davidson, R. J. (2004). Functional coupling of simultaneous electrical and metabolic activity in the human brain. Human Brain Mapping, 21(4), 257270. https://doi.org/10.1002/hbm.20004

Pascual-Marqui, R. D. (2002). Standardized low-resolution brain electromagnetic tomography (sLORETA): Technical details. Methods and Findings in Experimental and Clinical Pharmacology, 24(Suppl. D), 5-12.

Pizzagalli, D. A., Greischar, L. L., \& Davidson, R. J. (2003). Spatiotemporal dynamics of brain mechanisms in aversive classical conditioning: High-density event-related potential and brain electrical tomography analyses. Neuropsychologia, 41(2), 184-194. https://doi.org/10.1016/S0028-3932(02)00148-3

Pizzagalli, D. A., Nitschke, J. B., Oakes, T. R., Hendrick, A. M., Horras, K. A., Larson, C. L., ... Davidson, R. J. (2002). Brain electrical tomography in depression: the importance of symptom severity, anxiety, and melancholic features. Biological Psychiatry, 52(2), 73-85. https://doi.org/10.1016 /S0006-3223(02)01313-6

Pizzagalli, D., Pascual-Marqui, R. D., Nitschke, J. B., Oakes, T. R., Larson, C. L., Abercrombie, H. C., ... Davidson, R. J. (2001). Anterior cingulate activity as a predictor of degree of treatment response in major depression: Evidence from brain electrical tomography analysis. The American Journal of Psychiatry 158(3), 405-415. https://doi.org/10.1176/appi.ajp.158.3.405 
Rock, D. (2008). SCARF: A brain-based model for collaborating with and influencing others. NeuroLeadership Journal, 1, 1-9.

Rolls, E. T. (1999). The brain and emotion. New York, NY: Oxford University Press.

Thatcher, R. W. (2013). Latest developments in live $z$-score training: Symptom check list, phase reset, and LORETA $z-$ score biofeedback. Journal of Neurotherapy, 17(1), 69-87. https://doi.org/10.1080/10874208.2013.759032

The KEY Institute for Brain-Mind Research. (2014). Zürich, Switzerland: University of Zurich. Retrieved from http://www.uzh.ch/keyinst/

Sireci, S. G., \& Sukin, T. (2013). Test validity. In K. F. Geisinger, B. A. Bracken, J. F. Carlson, J.-I. C. Hansen, N. R. Kuncel, S. P. Reise, \& M. C. Rodriguez (Eds.), APA handbooks in psychology. APA handbook of testing and assessment in psychology, Vol. 1. Test theory and testing and assessment in industrial and organizational psychology (Ch. 4, pp. 61-84). Washington, DC: American Psychological Association. https://doi.org/10.1037/14047-004

To Neuromarkers And Beyond: Importance of Identifying Neuromarkers and Biomarkers to Predict and Improve Treatment Outcome for People with Developmental Trauma Ainat Rogel, Diana Martinez

Boston NeuroDynamics, Brookline, Massachusetts, USA

Developmental trauma (DT) or complex childhood trauma is arguably one of the most important public health challenges in the United States. It has a negative impact on the mental, physiological, and neurobiological functioning; leads to a lower quality of life or early death; and creates a substantial financial burden for the individuals affected, their families, and the healthcare system as a whole. Moreover, people with DT are often more resistant to traditional therapy. Research have shown that neurofeedback and biofeedback effectively treat people with DT. While most of the outcome of the research and of clinical treatment is based on subjective measurements, such as questionnaires, less is known about objective measurements, such as neuromarkers and biomarkers in predicting and quantitatively measuring outcome improvement due to the treatment. The goal of this presentation is to deepen the participants' understanding of DT and to address the ways to effectively collect and identify neuromarkers and biomarkers.

This presentation will highlight the importance of conducting neurological and physiological assessments (i.e., neuromarkers and physiomarkers), in addition to using traditional subjective assessments (e.g., questionnaires). At the individual level, we will explore ways of assessing clients' baseline functioning, providing guidance for treatment, and tracking progress. To further the use and improve the outcome of these techniques, we will discuss the benefits and challenges of identifying patterns of brain and physiological activities.

This presentation will begin by discussing ways to detect DT and how it differs from PTSD. After providing an overview of the impact of DT on health and well-being, it will focus on the brain development and functioning. Next, the presentation will identify several markers such as quantitative EEG (qEEG), event-related potential (ERP), and heart rate variability (HRV). We will continue by using these markers to track progress and measure the impact of the treatment at the individual level. Case presentations and clinical examples will be used to demonstrate these points. We will talk about implementation of the individual data to identify patterns of activities. These patterns can be used to predict the effectiveness of the treatment and to improve the outcome.

Overall, this workshop will provide participants with a more complete understanding of DT and ways to use neuromarkers and biomarkers to predict effectiveness of treatment and to improve treatment outcomes. We will finish with future directions and challenges to collect and identify these markers and what we can do as a community to collect and analyze this valuable data in order to understand DT and improve outcome of treatment.

\section{References}

Cook, A., Spinazzola, J., Ford, J., Lanktree, C., Blaustein, M., Cloitre, M., ... \& van der Kolk, B. (2005). Complex trauma in children and adolescents. Psychiatric Annals, 35(5), 390-398.

Huang-Storms, L., Bodenhamer-Davis, E., Davis, R., \& Dunn, J. (2006). QEEG-guided neurofeedback for children with histories of abuse and neglect: Neurodevelopmental rationale and pilot study. Journal of Neurotherapy, 10(4), 3-16. https://doi.org /10.1300/J184v10n04_02

Tan, G., Dao, T. K., Farmer, L., Sutherland, R. J., \& Gevirtz, R. (2011). Heart rate variability (HRV) and posttraumatic stress disorder (PTSD): A pilot study. Applied Psychophysiology and Biofeedback, 36(1), 27-35. https://doi.org/10.1007/s10484010-9141-y

Teicher, M. H., Andersen, S. L., Polcari, A., Anderson, C. M., Navalta, C. P., \& Kim, D. M. (2003). The neurobiological consequences of early stress and childhood maltreatment. Neuroscience \& Biobehavioral Reviews, 27(1-2), 33-44. https://doi.org/10.1016/s0149-7634(03)00007-1

The Effect of NeuroField Neurostimulation and Neuromdulation on Acute Opiate Detoxification Nicholas Dogris

NeuroField Neurotherapy, Inc., Santa Barbara, California, USA

Opiate addiction has been defined as a national epidemic in the United States according to a 2017 
report from the Centers of Disease Control and Prevention (CDCP). The rise of opioid deaths can be traced to three waves, with the first occurring in the 1990s when there was a significant increase in opioid prescriptions. The second wave occurred in 2010 when there was a spike in heroin deaths. The third wave occurred in 2013 when there was a significant increase in synthetic opioid deaths. According to the CDCP over 350,000 people have died from opioid overdoses from 1999 to 2017. The common method for medical detoxification from opioids is a 6-day taper in which the patient is given suboxone to reduce pain and cravings. During the 6-day taper, tDCS, tACS, and tRNS cranial stimulation were used and hypothesized to reduce cravings, anxiety, and depression and to improve sleep. The opioid group consisted of 53 patients. Pre- and post-EEGs were taken and analyzed showing significant changes in absolute power, coherence, and phase as compared to controls and neurofeedback only conditions. Furthermore, patient symptom ratings and PHQ9 and GAD7 surveys showed significant reduction in cravings, depression, and anxiety symptoms. No serious adverse side effects were reported in the stim-only condition. In this session, data will be presented showing the outcomes of this study.

\section{References}

Antonenko, D., Faxel, M., Grittner, U., Lavidor, M., \& Flöel, A. (2016). Effects of transcranial alternating current stimulation on Cognitive Functions in healthy young and older adults. Neural Plasticity, 2016, 4274127. https://doi.org/10.1155/2016 14274127

Bikson, M., Grossman, P., Thomas, C., Zannou, A. L., Jiang, J., Adnan, T., ... Woods, A. J. (2016). Safety of transcranial direct current stimulation: Evidenced based update 2016. Brain Stimulation, 9(5), 641-661. https://doi.org/10.1016 /j.brs.2016.06.004

Brunoni, A. R., Moffa, A. H., Fregni, F., Palm, U., Padberg, F., Blumberger, D., ... Loo, C. K. (2016) Transcranial direct current stimulation for acute major depressive episodes: Meta-analysis of individual patient data. The British Journal of Psychiatry, 208(6), 522-531. https://doi.org/10.1192/bjp.bp.115.164715

Palm, U., Chalah, M. A., Padberg, F., Al-Ani, T., Abdellaoui, M., Sorel, M., ... Ayache, S. S. (2016). Effects of transcranial random noise stimulation (tRNS) on affect, pain and attention in multiple sclerosis. Restorative Neurology and Neuroscience, 34(2), 189-199. https://doi.org/10.3233/RNN-150557

Van den Heuvel, M. P., \& Sporns, O. (2011). Rich-club organization of the human connectome. The Journal of Neuroscience, 31(44), 15775-15786. https://doi.org/10.1523 /JNEUROSCI.3539-11.2011
The Psychology of Adopting Medical Device Innovations in Mental Healthcare: The Case of Neurofeedback in the United States and the Netherlands

Mark Trullinger,

NeuroThrive, LLC, Silver Spring, Maryland, USA

Barriers to the acceptance of a medical device innovation, electroencephalogram neurofeedback (EEG-NFB) for the treatment of ADHD in children, were investigated with a mixed-method embedded design utilizing the theoretical frameworks of Latour and Rogers. Within Latour's framework EEG-NFB is a technological innovation that is part of a larger paradigm shift going on in medical healthcare treatment. Healthcare professionals act as "gatekeepers" to medical innovation within Roger's framework. Eighteen U.S. and Dutch healthcare professionals, who commonly diagnose and treat children with ADHD, participated in the study. The mixed-method design involved a semistructured interview embedded with quantitative assessments. The assessments included a self-monitoring questionnaire, selection and ranking of recommended treatments for a child with ADHD from a vignette, and identification and ranking of factors that influence the healthcare professional's decisionmaking process. Quantitative analyses were conducted with Tukey-Duckworth tests and a Spearman correlation. Qualitative analysis was conducted by word analysis with Leximancer. No significant differences were present between the U.S. and Dutch healthcare professionals. The sample were all highly aware of EEG-NFB with half being EEG-NFB practitioners, some who refer for EEG-NFB and some who were aware of EEG-NFB but did not refer patients for treatment or practice it themselves. EEG-NFB was one of the most commonly recommended treatments, and the highest ranked treatment, for the child with ADHD from the vignette. The main barrier identified was awareness about EEG-NFB as a treatment for ADHD in children. Technical knowledge about how to actually conduct, refer for, and evaluate progress in EEG-NFB was another major barrier. One of the recommendations to increase adoption is to initiate marketing campaigns focused on increasing awareness among healthcare professionals. Another recommendation is affordable or free continuing education courses for healthcare professionals targeted toward how to speak to a patient about the proposed mechanism of action for EEG-NFB, find a provider to refer to, and evaluate a patient's progress during a course of EEGNFB treatments. 


\section{References}

Denis, J.-L., Hébert, Y., Langley, A., Lozeau, D., \& Trottier, L.-H. (2002). Explaining diffusion patterns for complex health care innovations. Health Care Management Review, 27(3), 60-73. https://doi.org/10.1097/00004010-200207000-00007

Epocrates. (2016). Attention deficit hyperactivity disorder in children: Common Vignette 1. Retrieved from https://online.epocrates.com/diseases/14222/Attentiondeficithyperactivity-disorder-in-children/Common-Vignette

Epstein, R. M., Siegel, D. J., \& Silberman, J. (2008). Selfmonitoring in clinical practice: A challenge for medical educators. Journal of Continuing Education in the Health Professions, 28(1), 5-13. https://doi.org/10.1002/chp.149

European Commission. (2016). Joint-Statement on Mental Health for the EU Health Policy Platform on Mental Health and Policy. Retrieved from https://ec.europa.eu/health/sites/health /files/interest_groups/docs/ev_20161205_co02_en.pdf

HollandZorg. (2016). Alternative therapies and medicines. Ministry of Health, Government of the Netherlands. Retrieved from https://www.hollandzorg.com/dutch-healthcareinsurance/reimbursements/alternative-therapies-andmedicines

Latour, B. (1990). Technology is society made durable. The Sociological Review, 38(S1), 103-131. https://doi.org/10.1111 /j.1467-954X.1990.tb03350.x

Pigott, H. E., Cannon, R., \& Trullinger, M. (2018). The fallacy of sham-controlled neurofeedback trials: A reply to Thibault and Colleagues (2018). Journal of Attention Disorders. https://doi.org/10.1177/1087054718790802

Rogers, E. M. (2010). Diffusion of innovations (5th ed.). New York, NY: Free Press.

Smith, A. E., \& Humphreys, M. S. (2006). Evaluation of unsupervised semantic mapping of natural language with Leximancer concept mapping. Behavior Research Methods, 38(2), 262-279. https://doi.org/10.3758/BF03192778

Snyder, M. (1974). Self-monitoring of expressive behavior. Journal of Personality and Social Psychology, 30(4), 526537. https://doi.org/10.1037/h0037039

Thibault, R. T., \& Raz, A. (2017). The psychology of neurofeedback: Clinical intervention even if applied placebo. American Psychologist, 72(7), 679-688. https://doi.org /10.1037/amp0000118

Thibault, R. T., Veissière, S., Olson, J. A., \& Raz, A. (2018). Treating ADHD with suggestion: Neurofeedback and placebo therapeutics. Journal of Attention Disorders, 22(8), 707-711. https://doi.org/10.1177/1087054718770012

Tukey, J. W. (1959). A quick compact two sample test to Duckworth's specifications. Technometrics, 1(1), 31-48. https://doi.org/10.1080/00401706.1959.10489847

Van Doren, J., Arns, M., Heinrich, H., Vollebregt, M. A., Strehl, U., \& Loo, S. K. (2018). Sustained effects of neurofeedback in ADHD: A systematic review and meta-analysis. European Child \& Adolescent Psychiatry, 28(3), 293-305. https://doi.org /10.1007/s00787-018-1121-4

Walshe, K., \& Rundall, T. G. (2001). Evidence-based management: From theory to practice in health care. The Milbank Quarterly, 79(3), 429-457. https://doi.org/10.1111/1468-0009.00214

\section{An Integrative Neurotherapy Approach to Balancing the Gut-Brain Axis \\ Ashlie Bell \\ NeuroGrove Integrative Neurotherapy, Arvada, Colorado, USA}

Recent research within the fields of medicine, neuroscience, psychiatry, and microbiology has revealed a complex, remarkably interconnected relationship between the brain and gastrointestinal system. The gut-brain axis involves a number of complex feedback loops between the microbiome, intestinal barrier, mucosal immune system, neuroendocrine system, and hypothalamic-pituitaryadrenal (HPA) axis, as well as the enteric, autonomic, and central nervous systems (De Palma, Collins, Bercik, \& Verdu, 2014; Mayer, 2011). Through these communication channels, signals from the brain can modulate motor, sensory, and secretory functions of the gut, and signals from the gut can influence various aspects of psychological and cognitive function (De Palma, Vida, et al., 2014; Grenham, Clarke, Cryan, \& Dinan, 2011; Mayer, Knight, Mazmanian, Cryan, \& Tillisch, 2014). As such, alterations in one component can trigger a cascade of effects throughout the axis.

Exposure to prolonged or excessive stress-whether emotional trauma or pathogenic invasion-can produce especially detrimental effects on this axis, leading to chronic physical and psychological disorders (Bell \& Ross, 2014; Mayer et al., 2014). In fact, gut-brain imbalances have been implicated in a myriad of ailments, including anxiety, depression, PTSD, autism, attention deficits, eating disorders, irritable bowel syndrome, inflammatory bowel disease, Chron's disease, and ulcerative colitis, among others (Bischoff et al., 2014; Cryan \& Dinan, 2012; Mayer, 2011). Consequently, a failure to adequately address imbalances throughout this axis might inhibit clients' progress in neurotherapy.

This presentation will provide an overview of research on the gut-brain axis as it relates to the fields of neurofeedback and mental health. We will especially examine the impact of stress on the gut-brain axis, as well as the role of this axis in stress-related disorders. We will then discuss practical recommendations for an integrative neurotherapy approach to help clients effectively balance this axis, thus maximizing their physical, cognitive, and emotional well-being. This will include methods for reducing the brain's stress reactivity, balancing the neuroendocrine system, optimizing microbiota compositions, repairing the gut lining, restoring tight junctions in the blood-brain barrier, ensuring adequate nourishment, and reducing inflammation.

\section{References}

Bell, A., \& Ross, K. M. (2014). The neuro psycho physiological effects of chronic and excessive stress. American International Journal of Social Science, 3(1), 159-173. 
Bischoff, S. C., Barbara, G., Buurman, W., Ockhuizen, T., Schulzke, J.-D., Serino, M., ... Wells, J. M. (2014). Intestinal permeability-A new target for disease prevention and therapy. BMC Gastroenterology, 14, 189. https://doi.org /10.1186/s12876-014-0189-7

Cryan, J. F., \& Dinan, T. G. (2012). Mind-altering microorganisms: The impact of the gut microbiota on brain and behaviour. Nature Reviews Neuroscience, 13(10), 701-712. https://doi.org/10.1038/nrn3346

De Palman, G., Collins, S. M., Bercik, P., \& Verdu, E. F. (2014). The microbiota-gut-brain axis in gastrointestinal disorders: Stressed bugs, stressed brain or both? The Journal of Physiology, 592(14), 2989-2997. https://doi.org/10.1113 /jphysiol.2014.273995

De Palma, G., Vida, C., Santacruz, A., De Castro, N. M., De la Fuente, M., \& Sanz, Y. (2014). Impaired responses to gliadin and gut microbes of immune cells from mice with altered stress-related behavior and premature immune senescence. Journal of Neuroimmunology, 276(1), 47-57. https://doi.org /10.1016/j.jneuroim.2014.08.007

Grenham, S., Clarke, G., Cryan, J. F., \& Dinan, T. G. (2011). Braingut-microbe communication in health and disease. Frontiers in Physiology, 2, 94. https://doi.org/10.3389/fphys.2011.00094

Mayer, E. A. (2011). Gut feelings: The emerging biology of gutbrain communication. Nature Reviews Neuroscience, 12(8), 453-466. https://doi.org/10.1038/nrn3071

Mayer, E. A., Knight, R., Mazmanian, S. K., Cryan, J. F., \& Tillisch, K. (2014). Gut microbes and the brain: Paradigm shift in neuroscience. The Journal of Neuroscience, 34(46), 1549015496. https://doi.org/10.1523/JNEUROSCI.3299-14.2014

\section{Meta-Analysis of Mulitvariate Coherence Neurofeedback research \\ Robert Coben, Carl Armes \\ Integrated Neuroscience Services, Fayetteville, Arkansas, USA}

As the field of neurofeedback grows, a few novel neurofeedback approaches have emerged. While the use of functional magnetic resonance imaging (fMRI)based neuromodulation has shown efficacy as a form of treatment, $\mathrm{fMRI}$ is not cost effective or practical and yields low temporal resolution (Frey, Mühl, Lotte, \& Hachet, 2013). However, electroencephalography (EEG) is relatively inexpensive, practical, and yields high temporal resolution (Frey et al., 2013). Successful EEG-based neurofeedback therapy has been demonstrated in the treatment of clinical conditions and symptoms including decreasing symptoms related to seizure disorders (Sterman \& Egner, 2006) and learning disabilities (Coben, Wright, Decker, \& Morgan, 2015; Fernández et al., 2003). Additional findings have shown that EEG-based neurofeedback can reduce symptoms associated with autism spectrum disorder (Coben, 2013; Kouijzer, de Moor, Gerrits, Buitelaar, \& van Schie, 2009). Furthermore, a study by Wang et al. (2016) found that EEG characteristics associated with autism were reduced using prefrontal neurofeedback treatment. There is also evidence that the effects of these interventions last beyond the initial training period (Coben, 2013; Gevensleben et al., 2009).

Some researchers have found success using twochannel coherence training. However, the growing body of research concerning coherence assessment suggests that using a greater number of electrodes relative to the standard two-channel approach increases spatial acuity (Blinowska, 2011). Research has shown the use of four-channel multivariate coherence training is associated with significantly greater changes in power and coherence as compared to two-channel coherence training (Coben, Middlebrooks, Lightstone, \& Corbell, 2018). Advancing to four channels and calculating coherence metrics in a multivariate fashion led to greater changes in power by more than $50 \%$ and coherence by more than $400 \%$ (Coben et al., 2018).

This presentation will focus on the results of a metaanalysis on previous research conducted on several disorders whose subjects have received four-channel multivariate coherence training and were compared to an alternate treatment or comparison group. These include a mixed diagnosis study $(n=174)$, symptoms of autism $(n=110)$, mu suppression in autism ( $n=78)$, depression $(n=54)$, developmental trauma $(n=40)$, learning disabilities ( $n=63)$, epilepsy $(n=52)$, and traumatic brain injuries $(n=20)$ with a total sample size of $N=591$.

We plan to conduct this meta-analysis to help us understand the general impact of this form of neurofeedback which then may be compared to other types. Such a statistical approach will also help us understand if this form of neurofeedback has greater efficacy for certain conditions over others. We will also seek to understand if there are any mitigating factors such as medications, age, gender, or others. These findings will be presented and their implications discussed.

\section{References}

Blinowska, K. J. (2011). Review of the methods of determination of directed connectivity from multichannel data. Medical \& Biological Engineering \& Computing, 49(5), 521-529. https://doi.org/10.1007/s11517-011-0739-x

Coben, R. (2013). Neurofeedback for autistic disorders: Emerging empirical evidence. In M. F. Casanova, A. S. El-Baz, \& J. S. Suri (Eds.), Imaging the Brain in Autism (pp. 107-134). New York, NY: Springer. https://doi.org/10.1007/978-1-4614-684316

Coben, R., Middlebrooks, M., Lightstone, H., \& Corbell, M. (2018). Four channel multivariate coherence training: Development and evidence in support of a new form of neurofeedback. Frontiers in Neuroscience, 12, 729. https://doi.org/10.3389 /fnins.2018.00729 
Coben, R., Wright, E. K., Decker, S. L., \& Morgan, T. (2015). The impact of coherence neurofeedback on reading delays in learning disabled children: A randomized controlled study. NeuroRegulation, 2(4), 168-178. https://doi.org/10.15540 /nr.2.4.168

Fernández, T., Herrera, W., Harmony, T., Díaz-Comas, L., Santiago, E., Sánchez, L., ... Valdés, G. P. (2003). EEG and behavioral changes following neurofeedback treatment in learning disabled children. Clinical Electroencephalography, 34(3), 145-152. https://doi.org/10.1177/155005940303400308

Frey, J., Mühl, C., Lotte, F., \& Hachet, M. (2013). Review of the use of electroencephalography as an evaluation method for human-computer interaction. Proceedings from the International Conference on Physiological Computing Interface Workshop and Training Course (pp. 214-223), Lisbon, Portugal.

Gevensleben, H., Holl, B., Albrecht, B., Schlamp, D., Kratz, O., Studer, P., ... Heinrich, H. (2010). European Child \& Adolescent Psychiatry, 19(9), 715-724. https://doi.org/10.1007 /s00787-010-0109-5

Kouijzer, M. E. J., de Moor, J. M. H., Gerrits, B. J. L., Buitelaar, J. K., \& van Schie, H. T. (2009). Long-term effects of neurofeedback treatment in autism. Research in Autism Spectrum Disorders, 3(2), 496-501. https://doi.org/10.1016 /j.rasd.2008.10.003

Sterman, M. B., \& Egner, T. (2006). Foundation and practice of neurofeedback for the treatment of epilepsy. Applied Psychophysiology and Biofeedback, 31(1), 21. https://doi.org /10.1007/s10484-006-9002-X

Wang, Y., Sokhadze, E. M., El-Baz, A. S., Li, X., Sears, L., Casanova, M. F., \& Tasman, A. (2016). Relative power of specific EEG bands and their ratios during neurofeedback training in children with autism spectrum disorder. Frontiers in Human Neuroscience, 9, 723. https://doi.org/10.3389 /fnhum.2015.00723

\section{The Wisdom of Morphology}

\section{Tiff Thompson}

NeuroField Neurotherapy, Inc., Santa Barbara, California, USA

Reading the raw EEG is an artform that is an essential knowledge base of any practitioner using EEG to assess and diagnose their patients' conditions. Spindles, triangular shapes, sinusoidal, monomorphic, and archiform waveforms are just a few telling morphological signs that are imperative in understanding what is really going on. Does the waveform wax and wane? Does it travel in spindles or bursts? Does it appear only a few times in the record? What if it is rhythmic? These temporal dynamics are also imperative in a proper assessment of the person. When looking at the raw waveform, you will learn more than what any qEEG alone can tell you.

Through exploring the more insidious forms of artifact (i.e., electricity, channel noise, mixed metals, etc.) to detecting less commonly seen morphological forms in the EEG (i.e., lambda, mu, OIRDA, beta spindles, etc.), this lecture will guide the clinician through some of the more advanced ways of interpreting EEG so that the qEEG does not mislead one into misdiagnosis.

We are privileged to have many analysis and diagnostic tools to help us dissect, spatially and temporally analyze, condense, and summate the EEG into neat and tidy diagrams, but we fail our patients and our profession if we miss the devils in the details.

Finally, montages are necessary to understand the many ways in which we can assess and view the EEG. There is no best montage for all purposes. While linked ears can provide a global view, it is prone to contamination if there is a strong temporal finding or if there is contamination otherwise in the ear electrodes. Average and weighted average montages (such as the Laplacian and Hjorth montages), will highlight any local phenomena, and will uncover any significant temporal component, but will fail us to see global information. Bipolar montages are excellent for displaying phase reversals, which are indispensable in issues of head injury and seizure focus.

\section{References}

Chatrian, G. E., Bergamini, L., Dondey, M., Klass, D. W., Lennox, M., Buchthal, I., \& Petersen, I. (1974). A glossary of terms most commonly used by clinical electroencephalographers, Electroencephalography and Clinical Neurophysiology, 37, 538-548.

Nunez, P. L. (1995). Neocortical dynamics and human EEG rhythms. New York, NY: Oxford University Press.

Sanei, S., \& Chambers, J. A. (2007). EEG signal processing. Hoboken, NJ: John Wiley \& Sons, Inc.

\section{Cognitive Function Enhancement Through Photobiomodulation on Prefrontal Lobe and the EEG measurements \\ Li-da Huang, Kailin Wang \\ CytonSys, Lewes, Delaware, USA}

Brain functions have been proven to be affected by external stimuli. Photobiomodulation (PBM) using near-infrared is one of the effective ways to modulate the hemodynamic activities in the brain and to activate the key enzyme cytochrome c oxidase (CCO) in electron transport chain (ETC; Anders, Lanzafame, \& Arany, 2015; Anders et al., 2014; Barrett \& Gonzalez-Lima, 2013; Blanco, Saucedo, \& GonzalezLima, 2017; Rojas \& Gonzalez-Lima, 2017; Tian, Hase, Gonzalez-Lima, \& Liu, 2016; Vargas et al., 2017; Wang, Tian, Soni, Gonzalez-Lima, \& Liu, 2016). An 808-nm LED system with $250 \mathrm{~mW} / \mathrm{cm} 2$ power density, 10 min treatment on prefrontal area is 
adopted in this research. We investigated the cognitive effects of PBM using prefrontal cortex measures of attention, Psychomotor Vigilance Task (PVT), and memory, Delayed Match to Sample (DMS), to show the cognitive function enhancement. In addition, the primary brain activities as electroencephalography (EEG) is investigated to establish the link between the PBM and the EEG. In our preliminary results $(n=12)$, the $E E G$ related to the PBM on the subjects of various ages shows different responses in spectral and time domains, especially during the resting states. After PBM treatment, the peak-valley ratio of the waves in EEG has stronger contrast, and the effect lasts even the PBM has been stopped. This shows the PBM is not a short-term effect and could be observed in EEG. Our research indicates that the aging process and the brain function deterioration could be observed through the peak-valley ratio of the spectral-time measurements. Baseline vs. chronic (10 daily sessions, 10 min each) comparisons of mean cognitive scores all showed improvements, significant for PVT reaction time $(p<$ 0.001), PVT lapses $(p<0.001)$, and DMS correct responses $(p<0.05)$. The peak-valley ratio is enhanced about $3 \mathrm{~dB}$. This finding opens a window to investigate how the brain function enhancement is achieved at the system level and how the signaling enhancement are propagated.

\section{References}

Anders, J. J., Lanzafame, R. J., \& Arany, P. R. (2015). Low-level light/laser therapy versus photobiomodulation therapy. Photomedicine and Laser Surgery, 33(4), 183-184. https://doi.org/10.1089/pho.2015.9848

Anders, J. J., Moges, H., Wu, X., Erbele, I. D., Alberico, S. L., Saidu, E. K., ... Pryor, B. A. (2014). In vitro and in vivo optimization of infrared laser treatment for injured peripheral nerves. Lasers in Surgery and Medicine, 46(1), 34-45. https://doi.org /10.1002/lsm.22212

Barrett, D. W., \& Gonzalez-Lima, F. (2013). Transcranial infrared laser stimulation produces beneficial cognitive and emotional effects in humans. Neuroscience, 230, 13-23. https://doi.org /10.1016/j.neuroscience.2012.11.016

Blanco, N. J., Saucedo, C. L., \& Gonzalez-Lima, F. (2017). Transcranial infrared laser stimulation improves rule-based, but not information- integration, category learning in humans. Neurobiology of Learning and Memory, 139, 69-75. https://doi.org/10.1016/j.nlm.2016.12.016

Rojas, J. C., \& Gonzalez-Lima, F. (2017). Transcranial low-level laser (light) therapy for neurocognitive enhancement. In M. R. Hamblin, M. V. P. de Sousa, \& T. Agrawal (Eds.), Handbook of low-level laser therapy (1st ed., pp. 1057-1076). Singapore: Pan Stanford Publishing.

Tian, F., Hase, S. N., Gonzalez-Lima, F., \& Liu, H. (2016). Transcranial laser stimulation improves human cerebral oxygenation. Lasers in Surgery and Medicine, 48(4), 343-349. https://doi.org/10.1002/lsm.22471

Vargas, E., Barrett, D. W., Saucedo, C. L., Huang, L.-D., Abraham, J. A., Tanaka, H., ... Gonzalez-Lima, F. (2017). Beneficial neurocognitive effects of transcranial laser in older adults.
Lasers in Medical Science, 32(5), 1153-1162. https://doi.org/10.1007/s10103-017-2221-y

Wang, X., Tian, F., Soni, S. S., Gonzalez-Lima, F., \& Liu, H. (2016). Interplay between up-regulation of cytochrome-coxidase and hemoglobin oxygenation induced by nearinfrared laser. Scientific Reports, 6, 30540. https://doi.org/10.1038/srep30540

\section{POSTER PRESENTATIONS}

\section{Dosage of Low Frequency rTMS Affects Event- Related Potentials and Evoked and Induced $\mathbf{4 0}$ $\mathrm{Hz}$ Gamma Oscillations in Autism Spectrum Disorder \\ Estate Sokhadze, Manuel Casanova, Desmond Kelly, Eva Lamina, Emily Casanova \\ University of South Carolina School of Medicine-Greenville, Greenville, South Carolina, USA}

Background. Autism is defined as a spectrum of behavioral disorders that have in-common impairments in social interaction and communication skills, language deficits, and a restricted repertoire of interests and stereotyped activities. There are several theoretical models of the neuropathology of autism spectrum disorders (ASD), and one of them suggests the presence of an excessive cortical excitation/inhibition (E/I) ratio (Casanova, Buxhoeveden, \& Gomez, 2003; Rubenstein \& Merzenich, 2003) that affects functional connectivity. This model explains atypical event-related potential (ERP) and gamma oscillations observed in ASD during task performance (Sokhadze et al., 2009; Uzunova, Pallanti, \& Hollander, 2016).

Objectives. In our prior exploratory studies, we used different schedules of rTMS to investigate outcomes of rTMS in ASD. In this study, 124 high-functioning ASD children (IQ > 80, < 18 years of age) were recruited and assigned to either a waitlist group or one of three different number of weekly rTMS sessions (i.e., 6, 12, 18) to investigate effects of dosage on functional and behavioral outcomes. The project was aimed at selection of more effective length of rTMS course.

Methods. TMS consisted of trains of $1.0-\mathrm{Hz}$ pulses applied over dorsolateral prefrontal cortex. The experimental task was a three-stimulus visual oddball with illusory Kanizsa figures. Behavioral response variables included reaction time and error rate along with EEG indices such as ERP and evoked and induced gamma oscillations. One hundred and twelve patients completed the assigned number of rTMS sessions. 
Results. We found significant positive changes postTMS treatment in motor responses accuracy (percentage of committed errors, normative posterror slowing), in ERP indices and in evoked and induced gamma responses. Parental reports showed significant reductions in aberrant behavior scores as well as decreased scores of repetitive and stereotypic behaviors. The gains of outcomes increased with the total number of treatment sessions. Behavioral questionnaires (ABC, Aman \& Singh, 1994; RBS-R, Bodfish, Symons, \& Lewis, 1999; SRS-2, Constantino \& Gruber, 2005) showed significant improvements in ratings of autism symptoms both post-12- and 18session rTMS course. Results of our clinical research study showed most significant changes from baseline in functional measures of performance in oddball task and in behavioral symptom ratings following 18 sessions of rTMS treatment. Several measures showed a difference from baseline and waitlist in reaction time and ERP/EEG variables after 12 sessions of rTMS, but only a few of them reached statistical significance post-6-session rTMS course.

Conclusions. Our results suggest that rTMS, particularly after 18 sessions, facilitates cognitive control, attention, and target stimuli recognition by improving discrimination between task-relevant and task-irrelevant illusory figures in an oddball test. Improvement in executive functions and behavioral symptoms of autism further suggests that TMS has the potential to target core features of ASD. The results of this dosage-response study could serve as important prerequisites that could inform the planning of a blinded randomized clinical trial. Among potential implications should be considered combining rTMS with neurofeedback training aimed at reinforcement of neuromodulation effects using operant conditioning in similar manner as it was reported by our group earlier (Sokhadze et al., 2014).

\section{References}

Achenbach, T. M., \& Rescorla, L. A. (2012). Multicultural guide for the ASEBA forms and profiles for ages 11/2-18 (2nd ed.). Burlington, VT: University of Vermont.

Aman, M. G., \& Singh, N. N. (1994). Aberrant behavior checklistcommunity. Supplementary manual. East Aurora, NY: Slosson Educational Publications.

Bodfish, J. W., Symons, F. J., \& Lewis, M. H. (1999). Repetitive behavior scale. Morganton, NC: Western Carolina Center Research Reports.

Casanova, M. F., Buxhoeveden, D., \& Gomez, J. (2003). Disruption in the inhibitory architecture of the cell minicolumn: Implications for autism. The Neuroscientist, 9(6), 496-507. https://doi.org/10.1177/1073858403253552

Casanova, M. F., Sokhadze, E., Opris, I., Wang, Y., \& Li, X. (2015). Autism spectrum disorders: Linking neuropathological findings to treatment with transcranial magnetic stimulation. Acta
Paediatrica, $\quad$ 104(4), $\quad 346-355 . \quad$ https://doi.org/10.1111 lapa.12943

Constantino, J. N., \& Gruber, C. P. (2005). The social responsiveness scale (SRS): manual. Los Angeles CA: Western Psychological Services.

Rubenstein, J. L. R., \& Merzenich, M. M. (2003). Model of autism: Increased ratio of excitation/inhibition in key neural systems. Genes, Brain, and Behavior, 2(5), 255-267. https://doi.org /10.1034/j.1601-183X.2003.00037.x

Sokhadze, E. M., El-Baz, A., Baruth, J., Mathai, G., Sears, L., \& Casanova, M. F. (2009). Effects of low frequency repetitive transcranial magnetic stimulation (rTMS) on gamma frequency oscillations and event-related potentials during processing of illusory figures in autism. Journal of Autism and Developmental Disorders, 39(4), 619-634. https://doi.org/10.1007/s10803008-0662-7

Sokhadze, E. M., El-Baz, A. S., Tasman, A., Sears, L. L., Wang, Y., Lamina, E. V., \& Casanova, M. F. (2014). Neuromodulation integrating rTMS and neurofeedback for the treatment of autism spectrum disorder: An exploratory study. Applied Psychophysiology and Biofeedback, 39(3-4), 237-257. https://doi.org/10.1007/s10484-014-9264-7

Uzunova, G., Pallanti, S., \& Hollander, E. (2016). Excitatory/inhibitory imbalance in autism spectrum disorders: Implications for interventions and therapeutics. The World Journal of Biological Psychiatry, 17(3), 174-186. https://doi.org /10.3109/15622975.2015.1085597

\section{Incorporation of Neurofeedback to Improve} Sustained Attention During Learning Tasks Brooke Roberts, Laurel Keyes, Michael Hunter, Stephen Cruz, Neil McDonald, Walid Soussou

QUASAR, San Diego, California, USA

This project aimed to improve learning outcomes for students using an intelligent tutoring system (ITS) by employing electroencephalography (EEG)-based neurofeedback (NF). Our two-part hypothesis was 1) upregulating sensorimotor rhythm (SMR) increases a subject's ability to sustain attention for longer periods compared to a sham group and 2) the increase in sustained attention translates to improvements on cognitive tasks. Twenty-three adult subjects were recruited from the community to participate in six sessions, two or three times per week over a 3-week period. All subjects gave informed consent. Three subjects were unable to complete all six sessions and were removed from the study. Subjects were evenly divided between males and females. Subjects were randomly assigned to a NF protocol, either sham or active, and were evenly split between groups with 10 active and 10 sham. The study was double-blinded so that neither investigators nor subjects knew which protocol they received. Each session consisted of the NF protocol in addition to a battery of cognitive tasks, which were counterbalanced in order across sessions. The tasks consisted of single-digit column add (CA-1), triple-digit column add (CA-3), rotation tasks, and the Conners' Continuous Performance Test (CPT), intermixed with math tutorials presented 
by an ITS. The selected SMR-upregulation NF protocol (Vernon et al., 2003) has been reported to affect the SMR band, which is believed to contribute to sustained attention ability. The NF protocol was implemented using Mensia's Modulo software and Wearable Sensing's DSI-24 dry electrode EEG system. In this protocol, subjects were trained to fill in a jigsaw puzzle by raising the total power in their SMR band. Each training block lasted 3 min, across five blocks with a 10-s break between each block. Subjects were presented two indicators: a power bar on one side of the screen that fluctuated with the total SMR power and a jigsaw puzzle that filled in when the power met threshold requirements. Subjects were randomly assigned to one of two protocols. Group 1 was the active protocol, which used subjects' realtime EEG measurements; Group 2 was the sham condition, which used a random generator coupled with the subject's real-time noise metrics to keep the simulated training as realistic as possible. Results indicated that NF was effective at producing the targeted change in brain activity (upregulating SMR). Subjects in the active NF group also had significant increases in their sustained attention on the CPT task. Specifically, compared to the active group, the sham group had a slower reaction time by 9-70 ms (4-35\% change; $p<.009)$ and a greater change in omissions $(p<.01)$ on the learning task. Although we did not find any significant improvements in accuracy across the other cognitive tasks, we found that the active group missed between $6-26 \%$ fewer questions than the sham group $(p<.006)$ and responded faster $(p<.006)$ on the CA-3 task. In summary, while NF increased SMR activity and significantly improved sustained attention, performance on to learning and cognitive tasks were encouraging but not significant over this limited number of NF sessions.

\section{References}

Vernon, D., Egner, T., Cooper, N., Compton, T., Neilands, C., Sheri, A., \& Gruzelier, J. (2003). The effect of training distinct neurofeedback protocols on aspects of cognitive performance. International Journal of Psychophysiology, 47(1), 75-85. https://dx.doi.org/10.1016/S0167-8760(02)00091-0

*This material is based upon work supported by the National Science Foundation under Grant No. 1556096. Any opinions, findings, and conclusions or recommendations expressed in this material are those of the author(s) and do not necessarily reflect the views of the National Science Foundation.

\section{Neurofeedback: Behavioral and Neural} Changes in College Students with ADHD Rachel Bridges, Christopher Anzalone, Scott Decker University of South Carolina, Columbia, South Carolina, USA

Attention-deficit/hyperactivity disorder (ADHD) is a neurodevelopmental disorder characterized by a consistent presentation of inattentive, hyperactive, and/or impulsive behavior (American Psychiatric Association [APA], 2013). The subgroup of young adults with ADHD who pursue postsecondary education is of particular interest due to their enduring deficits in spite of relative academic achievement when compared to peers with the disorder who do not attend college (DuPaul, Weyandt, O'Dell, \& Varejao, 2009). Pharmaceutical treatments are among the most common strategies for mitigating symptoms of ADHD; however, due to trends in the sharing and illicit use of stimulant medication across universities, alternative treatment strategies that target the underlying neural mechanisms of the disorder are warranted. A meta-analysis concluded that neurofeedback (NF) treatment yields strong effect sizes, particularly for behavioral symptoms of ADHD (inattentiveness and impulsiveness; Arns, Conners, \& Kraemer, 2013). Moreover, the American Academy of Pediatrics (2011) listed NF as a promising treatment but highlighted the need for additional research.

The present proposal is part of one of the first investigations of NF that utilizes a randomized control design with a placebo condition in the treatment of college students with attention deficits. Moreover, the overarching aim of this study is to provide a better understanding of the utility of NF in the treatment of ADHD in college students, with the specific intention of exploring behavioral changes in the primary symptom domains of ADHD pre- and posttreatment. Additionally, exploratory analyses will be conducted to examine the relationship between behavioral presentations of the disorder and neural profiles. We hypothesize that students in the NF condition will demonstrate greater improvement on behavioralbased measures (according to symptoms outlined in the DSM-5; APA, 2013), as compared to students in the placebo condition. Additionally, we hypothesize that neural profiles, as measured by GEEG, will be associated with behavioral changes. Participants for the current study included undergraduate college students with a documented diagnosis of ADHD. Diagnostic status was confirmed by a trained doctoral-level graduate student using multimodal assessment procedures. Each student who met inclusion criteria completed a baseline qEEG. 
Students who did not display qEEG abnormalities consistent with ADHD (as measured by the Neuroguide symptom checklist) were excluded from the study, resulting in 16 participants who continued to the treatment phase. Participants were randomly assigned to the NF treatment condition $(n=8)$ and the placebo condition $(n=8)$. Treatment effects will be assessed by examining the changes in behavioral profiles using measures focused on primary symptom domains (i.e., inattentiveness and hyperactivity/impulsivity) between the baseline and posttreatment assessments. Additionally, the relationship between behavioral changes and neural profiles (as measured by pre- and posttreatment qEEG metrics) will be examined. Should significant differences be observed after participating in the NF treatment condition, it will be concluded that NF is a potentially viable tool for mitigating behavioral symptoms in university students with ADHD.

\section{References}

American Academy of Pediatrics. (2011). Neurofeedback, Hypnotherapy, and Guided Imagery. Retrieved from http://www.healthychildren.org/English/healthissues/conditions/adhd/Pages/Hypnotherapy-GuidedImagery-and-Biofeedback.aspx

American Psychiatric Association. (2013). Diagnostic and statistical manual of mental disorders: DSM-5 (5th ed.). Washington, DC: Author.

Arns, M., Conners, C. K., \& Kraemer, H. C. (2013). A decade of EEG theta/beta ratio research in ADHD: A meta-analysis. Journal of Attention Disorders, 17(5), 374-383. https://doi.org/10.1177/1087054712460087

DuPaul, G. J., Weyandt, L. L., O'Dell, S. M., \& Varejao, M. (2009). College students with ADHD: Current status and future directions. Journal of Attention Disorders, 13(3), 234-250. https://doi.org/10.1177/1087054709340650

\section{Neurofeedback: Performance-Based Profile Changes in the ADHD-Afflicted Brain Christopher Anzalone, Rachel Bridges, Scott Decker University of South Carolina, Columbia, South Carolina, USA}

While current prevalence rates for ADHD vary, it is estimated to affect five to eight percent of the general population across the lifespan (Goldstein, 2011). Though the characteristics of the disorder may change across development, symptoms of ADHD often continue to impair multiple functional domains of daily living.

Individuals with behavioral symptoms of ADHD often show deficits on performance-based measures (for a review see Woods, Lovejoy, \& Ball, 2002). Implementing compensatory strategies is the primary practice to improve overall prognosis. Since there is no cure for $A D H D$, interventions that target neural mechanisms of ADHD, other than psychiatric drugs, are in desperate need.

Research has clearly demonstrated the utility of EEG in diagnostic clinical evaluations for many neurological dysfunctions (Croona, Kihlgren, Lundberg, Eeg-Olofsson, \& Eeg-Olofsson, 1999; Leach, Stephen, Salveta, \& Brodie, 2006; McGonigal, Oto, Russell, Greene, \& Duncan, 2002; Mormann, Lehnertz, David, \& Elger, 2000; Thatcher, Walker, Gerson, \& Geisler, 1989). A new area of research, neurofeedback (NF), extends the functionality of EEG into a method of treatment. NF is one method that shows promise in treating neurodevelopmental conditions because it purportedly directly impacts brain functioning. Many research studies have generally supported the efficacy of NF for the treatment of neurodevelopmental disabilities in learning or attention (i.e., Breteler, Arns, Peters, Giepmans, \& Verhoeven, 2010; Gevensleben et al., 2009; Walker, 2010).

Although research studies support the use of neurofeedback, most published studies have methodological limitations. Namely, the American Academy of Pediatrics (2011) listed NF as promising but in need of more research. The current study uses a randomized control research design with a placebo (or sham) condition for the treatment of children with learning and attention problems. By overcoming the methodological limitations of past research, this study will provide a better understanding of the effectiveness of NF. The goal of this study is to test the effects of NF in college students who experience functional impairment as a result of ADHD, with the underlying hypothesis that students in the NF condition will demonstrate greater improvement on performance-based measures (i.e., CPT, BG-II, and WJ III subtests) than students in the sham condition.

Participants included 16 college undergraduates who were documented as having a diagnosis of ADHD. Each potential participant completed a baseline qEEG; using the Neuroguide symptoms checklist, only those students who exhibited qEEG abnormalities consistent with ADHD were permitted to continue in the study $(N=16)$.

Treatment effects will be assessed by examining the changes in performance on the neurocognitive measures (i.e., CPT, BG-II, and WJ III subtests) between the baseline and posttreatment assessments. We expect to see significant improvements between pre- and post-NF cognitive measures in the treatment group, but stable 
performance in the sham group. Should significant changes be observed after receiving NF training, we will conclude that NF has the potential to improve cognitive functioning in college students with ADHD, and thus postulate that NF may be a viable treatment option to improve the prognosis for individuals afflicted with ADHD.

\section{References}

American Academy of Pediatrics. (2011). Neurofeedback, Hypnotherapy, and Guided Imagery. Retrieved from http://www.healthychildren.org/English/healthissues/conditions/adhd/Pages/Hypnotherapy-GuidedImagery-and-Biofeedback.aspx

Breteler, M. H. M., Arns, M., Peters, S., Giepmans, I., \& Verhoeven, L. (2010). Improvements in spelling after QEEG-based neurofeedback in dyslexia: A randomized controlled treatment study. Applied Psychophysiology and Biofeedback, 35(1), 511. https://doi.org/10.1007/s10484-009-9105-2

Croona, C., Kihlgren, M., Lundberg, S., Eeg-Olofsson, O., \& EegOlofsson, K. E. (1999). Neuropsychological findings in children with childhood epilepsy with centrotemporal spikes. Developmental Medicine \& Child Neurology, 41(12), 813-818. https://doi.org/10.1111/j.1469-8749.1999.tb00547.x

Gevensleben, H., Holl, B., Albrecht, B., Vogel, C., Schlamp, D., Kratz, O., ... Heinrich, H. (2009), Is neurofeedback an efficacious treatment for ADHD? A randomized controlled clinical trial. Journal of Child Psychology and Psychiatry, 50(7), 780-789. https://doi.org/10.1111/j.1469-7610.2008.02033.x

Goldstein, S. (2011). Attention-deficit/ hyperactivity disorder. In S. Goldstein \& C. R. Reynolds (Eds.), Handbook of Neurodevelopmental and Genetic Disorders in Children (2nd ed.). New York, NY: The Guilford Press.

Leach, J. P., Stephen, L. J., Salveta, C., \& Brodie, M. J. (2006). Which electroencephalography (EEG) for epilepsy? The relative usefulness of different EEG protocols in patients with possible epilepsy. Journal of Neurology, Neurosurgery \& Psychiatry, 77(9), 1040-1042. https://doi.org/10.1136 /jnnp.2005.084871

McGonigal, A., Oto, M., Russell, A. J. C., Greene, J., \& Duncan, R. (2002). Outpatient video EEG recording in the diagnosis of non-epileptic seizures: A randomised controlled trial of simple suggestion techniques. Journal of Neurology, Neurosurgery \& Psychiatry, 72(4), 549-551. https://doi.org/10.1136 /jnnp.72.4.549

Mormann, F., Lehnertz, K., David, P., \& Elger, C. E. (2000). Mean phase coherence as a measure for phase synchronization and its application to the EEG of epilepsy patients. Physica D: Nonlinear Phenomenon, 144(3-4), 358-369. https://doi.org /10.1016/S0167-2789(00)00087-7

Thatcher, R. W., Walker, R. A., Gerson, I., \& Geisler, F. H. (1989). EEG discriminant analyses of mild head trauma. Electroencephalography and clinical neurophysiology, 73(2), 94-106. https://doi.org/10.1016/0013-4694(89)90188-0

Walker, J. E. (2010). Recent advances in quantitative EEG as an aid to diagnosis and as a guide to neurofeedback training for cortical hypofunctions, hyperfunctions, disconnections, and hyperconnections: Improving efficacy in complicated neurological and psychological disorders. Applied Psychophysiology and Biofeedback, 35, 25-27. https://doi.org /10.1007/s10484-009-9107-0

Woods, S. P., Lovejoy, D. W., \& Ball, J. D. (2002). Neuropsychological characteristics of adults with ADHD: a comprehensive review of initial studies. The Clinical Neuropsychologist, 16(1), 12-34. https://doi.org/10.1076 /clin.16.1.12.8336
Pilot Study of the Efficacy of Mobile

Neurofeedback for Attention-

Deficit/Hyperactivity Disorder (ADHD)

Glen M. Doniger, Aziz Kaddan

Myndlift, Tel Aviv, Israel

Background. Neurofeedback is commonly regarded as an adjunctive treatment for ADHD, and randomized controlled trials have shown significant benefit attributable to this intervention (Arns, Ridder, Strehl, Breteler, \& Coenen, 2009; Lofthouse, Arnold, Hersch, Hurt, \& DeBeus, 2012; Micoulaud-Franchi et al., 2014). The present study is a controlled trial to evaluate the specific benefit of neurofeedback training using Myndlift, a clinician-guided wearable, mobile neurofeedback system.

Methods. Nineteen participants (all male, ages 815) diagnosed with ADHD were recruited. The intervention group $(n=12)$ engaged in theta/beta neurofeedback training three to four times per week for 9 weeks, totaling an average of 21 sessions per participant. The control group $(n=7)$ did not receive any treatment during the 9-week period. Participants in both groups were assessed with a computerized continuous performance test (CPT; MOXO, Neuro Tech Solutions Ltd.) before and after the 9-week period. During the CPT, participants were to respond to target stimuli and ignore nontarget stimuli in the presence of visual and auditory distracters. Outcomes were age- and gender-adjusted $z$-scores for overall (total) performance, attention, timeliness, impulsivity, and hyperactivity. The CPT assessment report indicated a performance level for each obtained score as follows: good (1), standard (2), weak (3), or difficulty in performance (4). The report further classified performance levels $1-3$ as "within the norm" and performance level 4 as "outside the norm."

Results. Sixteen participants completed the study ( $n$ $=10$ in the intervention group; $n=6$ in the control group). The intervention group showed significant improvement in overall performance, attention, inhibition, and hyperactivity $(p<.008)$. In contrast, the control group did not show significant improvement. Change from 0 to 9 weeks was significantly greater in the intervention group for overall performance and attention $(p=.030)$. Overall performance level was improved for $90 \%$ of neurofeedback participants as compared with $34 \%$ of controls. Knowledge of whether the participant was assigned to the intervention or control group improved prediction of change in overall CPT performance level by $70 \%(p<.001)$. For all 
outcomes, a greater percentage of participants in the intervention group improved in performance level compared with controls. Change in performance level was significantly greater for the intervention group compared with controls for overall performance $(p=.018)$ and attention $(p=.048)$. For all outcomes, median change in performance level for the passive control group was 0 , reflecting no change. Finally, compared with controls, more participants were reclassified as "within the norm" following neurofeedback. The difference was most salient for overall performance- 8 of the 10 neurofeedback participants were reclassified as normal compared with one of the six control participants.

Conclusions. This controlled study provides encouraging evidence for the efficacy of the Myndlift home-based clinician-guided neurofeedback system. In a small cohort of children with ADHD, a 9-week neurofeedback training protocol consistently improved performance on objective measures of ADHD symptomatology. Follow-up randomized controlled trials including active control conditions in larger cohorts are needed to further establish the efficacy of the tool.

\section{References}

Arns, M., deRidder, S., Strehl, U., Breteler, M., \& Coenen, A. (2009). Efficacy of neurofeedback treatment in ADHD: The effects on inattention, impulsivity and hyperactivity: A metaanalysis. Clinical EEG and Neuroscience, 40(3), 180-189. https://doi.org/10.1177/155005940904000311

Lofthouse, N., Arnold, L. E., Hersch, S., Hurt, E., \& DeBeus, R. (2012). A review of neurofeedback treatment for pediatric ADHD. Journal of Attention Disorders, 16(5), 351-372. https://doi.org/10.1177/1087054711427530

Micoulaud-Franchi, J.-A., Geoffroy, P. A., Fond, G., Lopez, R., Bioulac, S., \& Philip, P. (2014). EEG neurofeedback treatments in children with ADHD: An updated meta-analysis of randomized controlled trials. Frontiers in Human Neuroscience, $\quad 8, \quad 906 . \quad$ https://doi.org/10.3389 /fnhum.2014.00906

\section{Predictors of Neurofeedback Outcomes} Following qEEG Individualized Protocols for Anxiety

Jessica Gregory, Devon Romero, Mark Jones

The University of Texas at San Antonio, San Antonio, Texas, USA

According to the National Institute of Mental Health (NIHM), anxiety disorders are a prevalent mental health concern affecting $19.1 \%$ of adults in the United States (NIMH, 2017). A majority of Americans experience stress during their lifetime; however, anxiety disorders are pervasive and disrupt daily functioning. Neurofeedback, a form of biofeedback, has displayed positive outcomes for reducing anxiety symptoms (Dreis et al., 2015).

The current study uses a within-subjects research design which included qEEG-individualized neurofeedback protocols for anxiety. Protocols were determined by comparing a client's pretest qEEG data to a normative database, and the resulting data was used to develop an individualized protocol. Feedback consisted of games, sounds, animations, and analogical presentations. In addition, the Zung Self-Rating Anxiety Scale for adults, Screen for Anxiety-Related Disorders (SCARED) for children, and Achenbach System of Empirically Based Assessment (ASEBA) were used as self-report assessments for anxiety during pre, post, and followup qEEG sessions. Clients were recruited from San Antonio and the surrounding area to receive counseling and neurofeedback treatment free of charge. Clients were asked to attend biweekly neurofeedback sessions for which they agreed to attend a minimum total of 15 training sessions. The neurofeedback training sessions were administered by clinical mental health counseling students and counselor education doctoral students.

The purpose of the current study is to use multilevel modeling to determine predictors of neurofeedback outcomes following individualized neurofeedback anxiety protocols. For this study, a two-level growth model was constructed to identify predictors of outcomes. The times of measurement were level-1 and individual client characteristics were level-2. The individual growth trajectories were fitted in the level-1 model. Outcome scores from the qEEG and other identified instruments were modeled as a function of time. Data will be presented from research findings and implications for neurofeedback will be discussed. In addition, implications for future research and counseling will be discussed.

\section{References}

Dreis, S. M., Gouger, A. M., Perez, E. G., Russo, G. M., Fitzsimmons, M. A., \& Jones, M. S. (2015). Using neurofeedback to lower anxiety symptoms using individualized qEEG protocols: A pilot study. NeuroRegulation, 2(3), 137148. https://doi.org/10.15540/nr.2.3.137

National Institute of Mental Health (2017). Any Anxiety Disorder. Retrieved from https://www.nimh.nih.gov/health/statistics/anyanxiety-disorder.shtml\#part_155094 
Psychophysiological Correlates of Prefrontal Neurofeedback in Children with Autism

Spectrum Disorder: Pilot Study

Estate Sokhadze ${ }^{1}$, Desmond Kelly², Meghan Doyle ${ }^{2}$, Manuel Casanova ${ }^{1}$

${ }^{1}$ University of South Carolina School of Medicine-Greenville, Greenville, South Carolina, USA

${ }^{2}$ Greenville Health System, Greenville, South Carolina, USA

Background. Neurofeedback is one of the most promising methods for training EEG self-regulation in children with autism spectrum disorder (ASD). There are several neurofeedback protocols proposed for ASD with most differences being in the type of training (e.g., theta/beta ratio, coherence), topography ( $\mathrm{Cz}$ or $\mathrm{Pz}$ ), guidance by quantitative EEG (qEEG) and number of sessions (e.g., 20 vs. 30). Most studies of neurofeedback in ASD focus on behavioral and EEG outcomes and do not analyze associated psychophysiological processes taking place during successful training. In particular, some cardiorespiratory and electrodermal effects of training are important for the understanding of neurofeedback training effects and defining their role as potential moderators.

Objectives. We proposed that 24 sessions of prefrontal neurofeedback training will be accompanied by changes in power of targeted EEG bands (e.g., $40 \mathrm{~Hz}$-centered gamma band) and ratios of individuals bands (e.g., theta/beta ratio), as well as by changes in electrodermal and cardiorespiratory indices. Autonomic activity patterns were hypothesized to reflect specifics of psychophysiological processes occurring during neurofeedback training in children with autism (Sokhadze, Casanova, Casanova, Klusek, \& Roberts, 2019).

Methods. Outcomes measures along with EEG, ECG, pneumogram, and skin conductance measures included behavioral ratings by parents. The protocol used a training for wide-band EEG amplitude suppression ("InhibitAll") with simultaneous upregulation of the relative power of $40 \mathrm{~Hz}$-centered gamma subband activity. In a pilot study on six children diagnosed with ASD (13.6 years, $S D=1.3$, two girls) in a 24-session course aimed at prefrontal $40 \mathrm{~Hz}$-centered EEG gamma upregulation and thetato-beta ratio downregulation we recorded ECG, pneumogram, and electodermal activity. For each session of neurofeedback, qEEG analysis at the training site was completed to determine the relative power of the individual bands (theta, beta, and gamma) and their ratios (theta/beta) within and between sessions. We analyzed Aberrant Behavior
Checklist (ABC; Aman \& Singh, 1994) and ASEBA (Achenbach \& Rescorla, 2012) ratings by parents (pre- and posttreatment).

Results. Dynamics of psychophysiological measures were analyzed during each neurofeedback session and across the whole course. Regression analysis revealed significant linear increase of skin conductance level (SCL, $p=0.002$ ) along with decrease of respiration rate (RSR, $p<.001)$ during each neurofeedback session without any statistical changes of SCL of RSR across the course of training. Heart rate variability (HRV) measures (e.g., HF of HRV) showed significant increase during each individual session. According to parental reports hyperactivity subscale scores of $\mathrm{ABC}(p=.024)$ and ASEBA DSM-oriented scores of attention-related problems $(p=.014)$ decreased by the end of neurofeedback course.

Conclusions. Psychophysiological measures represent useful markers of attention and emotional engagement of children with ASD during neurofeedback and can be used as predictors of successful performance during training sessions and general behavioral outcome of the intervention. In particular, trend towards increase of electrodermal activity along with RSR deceleration and increased indices of respiratory sinus arrhythmia in HRV may reflect more active attention to training targets and/or experience of emotion states reflected in the observed pattern of psychophysiological indices.

\section{References}

Achenbach, T. M., \& Rescorla, L. A. (2012). Multicultural guide for the ASEBA forms and profiles for ages 11/2-18 (2nd ed.). Burlington, VT: University of Vermont, Research Center for Children, Youth, \& Families.

Aman, M. G., \& Singh, N. N. (1994). Aberrant behavior checklistcommunity. Supplementary manual. East Aurora, NY: Slosson Educational Publications.

Sokhadze, E. M., Casanova, M. F., Casanova, E. L., Klusek, J., \& Roberts, J. (2019). Autonomic nervous system dysfunctions in children with autism spectrum disorder. In E. M. Sokhadze \& M. F. Casanova (Eds.), Autism Spectrum Disorder: Neuromodulation, Neurofeedback and Sensory Integration Approaches to Research and Treatment (pp. 169-206). Murfreesboro, TN, FNNR.

Stuttering Throughout Development:

A Case Series of Neuromodulation Patients

Charles Wasserman, Robert Turner

Network Neurology LLC, Charleston, South Carolina, USA

Stuttering has been found to affect approximately $5 \%$ of preschool-aged children and an overall $1 \%$ of the 
general population (Chang, Garnett, Etchell, \& Chow, 2018; Kent, 2000). Known by several names (e.g., childhood-onset fluency disorder, developmental stuttering), the cause of stuttering has been, and still is, the subject of a lively debate among researchers. While the primary theory of etiology is that stuttering is the result of dysregulation between auditory processing and motor areas of the brain (Kent, 2000; Postma, Kolk, \& Povel, 1990), there are still others who view stuttering as a disorder of speech motor control specifically (Einarsdóttir \& Ingham, 2008), and yet further research has indicated that the basal ganglia also play a significant role in the disorder (Craig-McQuaide, Akram, Zrinzo, \& Tripoliti, 2014). Many different therapeutic interventions have been researched for stuttering, including behavioral therapy (Ingham, Ingham, Euler, \& Neumann, 2018) and deep brain stimulation (Craig-McQuaide et al., 2014), and there is even a growing literature on neurofeedback (Bingham, 2013). In 2004, Özge and colleagues found increased delta activity in stutterers vs. age-sex matched controls (especially in rightfrontal brain regions; Özge, Toros, \& Çömelekoğlu, 2004). Similarly, a 2008 paper by Dr. Ratcliff-Baird showed an increase in frontal theta in stutterers compared to a group of controls. The same study also found a deficit in alpha (Ratcliff-Baird, 2008) and suggested that since similar EEG phenotypes of ADHD had been shown to respond well to neurofeedback (Monastra, 2005) that using neurofeedback as an intervention for stuttering would be worth pursuing. In this study, we present a case series of four neurofeedback patients who were seen for developmental stuttering ranging in age from 8 to 20 years old. All four of the patients had positive outcomes from neurofeedback paired with a wholeperson approach (including nutrition, sleep-habits, and interaction with digital technology). For each patient qEEG analysis was performed on eyes-open and eyes-closed baseline data both before and after neurofeedback therapy, and all patients reported a positive improvement in symptoms.

\section{References}

Bingham, B. (2013). Multi-modal treatment of stuttering: A case study showing neurofeedback coupled with traditional speech therapy. ISNR 20th Annual Conference. International Society for Neurofeedback and Research, Orlando, FL.

Chang, S.-E., Garnett, E. O., Etchell, A., \& Chow, H. M. (2018). Functional and neuroanatomical bases of developmental stuttering: Current insights. The Neuroscientist, 107385841880359 . https://doi.org/10.1177 $/ 1073858418803594$

Craig-McQuaide, A., Akram, H., Zrinzo, L., \& Tripoliti, E. (2014). A review of brain circuitries involved in stuttering. Frontiers in Human Neuroscience, 8, 884. https://doi.org/10.3389 /fnhum.2014.00884
Einarsdóttir, J., \& Ingham, R. J. (2008). The effect of stuttering measurement training on judging stuttering occurrence in preschool children who stutter. Journal of Fluency Disorders, 33(3), 167-179. https://doi.org/10.1016/j.jfludis.2008.05.004

Ingham, R. J., Ingham, J. C., Euler, H. A., \& Neumann, K. (2018). Stuttering treatment and brain research in adults: A still unfolding relationship. Journal of Fluency Disorders, 55, 106119. https://doi.org/10.1016/j.jfludis.2017.02.003

Kent, R. D. (2000). Research on speech motor control and its disorders: A review and prospective. Journal of Communication Disorders, 33(5), 391-428. https://doi.org /10.1016/S0021-9924(00)00023-X

Monastra, V. J. (2005). Electroencephalographic biofeedback (neurotherapy) as a treatment for attention deficit hyperactivity disorder: Rationale and empirical foundation. Child and Adolescent Psychiatric Clinics of North America, 14(1), 55-82. https://doi.org/10.1016/j.chc.2004.07.004

Özge, A., Toros, F., \& Çömelekoğlu, Ü. (2004). The role of hemispheral asymmetry and regional activity of quantitative EEG in children with stuttering. Child Psychiatry and Human Development, 34(4), 269-280. https://doi.org/10.1023 /B:CHUD.0000020679.15106.a4

Postma, A., Kolk, H., \& Povel, D.-J. (1990). On the relation among speech errors, disfluencies, and self-repairs. Language and Speech, 33(1), 19-29. https://doi.org/10.1177 1002383099003300102

Ratcliff-Baird, B. (2008). ADHD and stuttering: Similar EEG profiles suggest neurotherapy as an adjunct to traditional speech therapies. Journal of Neurotherapy, 5(4), 5-22. https://doi.org /10.1300/J184v05n04_02

\section{The Effect of Thermal Biofeedback on}

Migraine, Disability, and Quality of Life in an Underserved Pediatric Neurology Population Karen McKearney

New York Presbyterian Medical Center/Columbia University, New York, New York, USA

Headache is the most common disabling neurological disorder, with migraine affecting an estimated 1 in 10 school-age children and directly costing around $\$ 1$ billion per year (Bellini et al., 2013; DeMaagd, 2008; $\mathrm{Hu}$, Markson, Lipton, Stewart, \& Berger, 1999). Pediatric migraine affects quality of life resulting in greater risk of impaired physical health, school absenteeism, decreased involvement in peer activities, and altered relationships with family and friends (Stubberud, Varkey, McCrory, Pedersen, \& Linde, 2016). Depression and anxiety are prevalent comorbid conditions in migraineurs, and symptoms are positively correlated with headache frequency (Chu et al., 2018). The need for a nonpharmacologic approach to headache treatment in children stems from concerns about prevention headache medication and reluctance to take them, side effects, and poor efficacy (Powers et al., 2017). Biofeedback therapy, an effective treatment for headaches, teaches a patient how to control their body responses to pain and stress, the most frequently reported migraine trigger (Stubberud et al., 2016). Our prospective, randomized, wait-listed controlled study 
population is comprised of children and adolescents (ages 8-21) recruited from general pediatric and neurology clinics. To qualify, participants must experience at least four migraine headaches a month using the International Headache Society (IHS) criteria and be Medicaid/Medicaid managed care plan insured. The purpose of this trial is to determine if thermal biofeedback therapy is more effective than the standard headache treatment in improving headache frequency, duration, intensity, disability, and quality of life. The waitlist control group will be given the standard of care headache treatment, lifestyle modification education, acute and preventative medication treatments, and routine follow-up. In addition, the treatment group will undergo eight weekly 20-min biofeedback sessions. Data will be compared between the biofeedback and control groups, obtained using a paired $t$-test of headache diary, the Pediatric Migraine Disability Assessment (PedMIDAS), and the Pediatric Quality of Life Index (PedsQL) questionnaire. Subjects who receive thermal biofeedback therapy are hypothesized to demonstrate short- and long-term improvements in headache characteristics, quality of life, and migraine disability. We will discuss the preliminary results comparing data in both groups from baseline appointment, final session, 1-month follow-up, and 3-month follow-up.

\section{References}

Bellini, B., Arruda, M., Cescut, A., Saulle, C., Persico, A., Carotenuto, M., ... Guidetti, V. (2013). Headache and comorbidity in children and adolescents. The Journal of Headache and Pain, 14, 79. https://doi.org/10.1186/11292377-14-79

Chu, H.-T., Liang, C.-S., Lee, J.-T., Yeh, T.-C., Lee, M.-S., Sung, Y.-F., \& Yang, F.-C. (2018). Associations between depression/anxiety and headache frequency in migraineurs: A cross-sectional study. Headache, 58(3), 407-415. https://doi.org/10.1111/head.13215

DeMaagd, G. (2008). The pharmacological management of migraine, part 1: Overview and abortive therapy. $P \& T, 33(7)$, 404-416.

Hu, X. H., Markson, L. E., Lipton, R. B., Stewart, W. F., \& Berger, M. L. (1999). Burden of migraine in the United States: Disability and economic costs. Archives of Internal Medicine, 159(8), 813-818. https://doi.org/10.1001/archinte.159.8.813

Powers, S. W., Coffey, C. S., Chamberlin, L. A., Ecklund, D. J., Klingner, E. A., Yankey, J. W., ... Hershey, A. D. (2017). Trial of amitriptyline, topiramate, and placebo for pediatric migraine. The New England Journal of Medicine, 376(2), 115-124. https://doi.org/10.1056/NEJMoa1610384

Stubberud, A., Varkey, E., McCrory, D. C., Pedersen, S. A., \& Linde, M. (2016). Biofeedback as prophylaxis for pediatric migraine: A meta-analysis. Pediatrics, 138(2), e20160675. https://.doi.org/10.1542/peds.2016-0675

Received: November 24, 2019

Accepted: November 24, 2019

Published: December 12, 2019 